\title{
Functional Morphology of the Fin Rays of Teleost Fishes
}

\author{
Brooke E. Flammang, ${ }^{1 *}$ Silas Alben, ${ }^{2}$ Peter G.A. Madden, ${ }^{1}$ and George V. Lauder ${ }^{1}$ \\ ${ }^{1}$ Department of Organismic and Evolutionary Biology, Harvard University, Cambridge, Massachusetts 02138 \\ ${ }^{2}$ Department of Mathematics, University of Michigan, Ann Arbor, Michigan 48109
}

ABSTRACT Ray-finned fishes are notable for having flexible fins that allow for the control of fluid forces. A number of studies have addressed the muscular control, kinematics, and hydrodynamics of flexible fins, but little work has investigated just how flexible ray-finned fish fin rays are, and how flexibility affects their response to environmental perturbations. Analysis of pectoral fin rays of bluegill sunfish showed that the more proximal portion of the fin ray is unsegmented while the distal $60 \%$ of the fin ray is segmented. We examined the range of motion and curvatures of the pectoral fin rays of bluegill sunfish during steady swimming, turning maneuvers, and hovering behaviors and during a vortex perturbation impacting the fin during the fin beat. Under normal swimming conditions, curvatures did not exceed $0.029 \mathrm{~mm}^{-1}$ in the proximal, unsegmented portion of the fin ray and $0.065 \mathrm{~mm}^{-1}$ in the distal, segmented portion of the fin ray. When perturbed by a vortex jet traveling at approximately $1 \mathrm{~ms}^{-1}(67 \pm 2.3 \mathrm{mN}$ s.e. of force at impact), the fin ray underwent a maximum curvature of $9.38 \mathrm{~mm}^{-1}$. Buckling of the fin ray was constrained to the area of impact and did not disrupt the motion of the pectoral fin during swimming. Flexural stiffness of the fin ray was calculated to be $565 \times 10^{-6} \mathrm{Nm}^{2}$. In computational fluid dynamic simulations of the fin-vortex interaction, very flexible fin rays showed a combination of attraction and repulsion to impacting vortex dipoles. Due to their small bending rigidity (or flexural stiffness), impacting vortices transferred little force to the fin ray. Conversely, stiffer fin rays experienced rapid small-amplitude oscillations from vortex impacts, with large impact forces all along the length of the fin ray. Segmentation is a key design feature of ray-finned fish fin rays, and may serve as a means of making a flexible fin ray out of a rigid material (bone). This flexibility may offer intrinsic damping of environmental fluid perturbations encountered by swimming fish. J. Morphol. 274:1044-1059, 2013. (ㅇ 2013 Wiley Periodicals, Inc.

KEY WORDS: perturbation; flexural stiffness; structural properties; segment; curvature

Contract grant sponsor: ONR; contract grant number: N0001409-1-0352; Contract grant sponsor: ONR-MURI; contract grant number: N00014-03-1-0897; Contract grant sponsor: NSF; contract grant numbers: EFRI-0938043; DMS-1022619; Contract grant sponsor: Sloan Research Fellowship (to SA).

*Correspondence to: Brooke E. Flammang, Department of Organismic and Evolutionary Biology, Harvard University, Cambridge, MA 02138. E-mail: bflammang@post.harvard.edu

\section{INTRODUCTION}

Bluegill sunfish, like many bony fish, swim by moving and changing the shape of their very flexible fins via muscular control of the lepidotrichia (Walker, 2004b; Lauder et al., 2006; Alben et al., 2007; Lauder and Madden, 2007; Chadwell et al., $2012 a, b)$. Each lepidotrich is made up of two hemitrichs (Geerlink and Videler, 1987; Videler, 1993; Taft and Taft, 2012); the hemitrichs are controlled individually by muscles at their base which control curvature and stiffness of each fin ray (Alben et al., 2007). Fin rays are flexible, yet are formed from acellular bone (Moss, 1963) which is more mineralized than the cellular bone in the skeleton, but has a similar Young's modulus (Cohen et al., 2012). Flexible fin rays, or lepidotrichia, are a hallmark of bony fishes; sister hemitrichs (half rays) composed of a proximal unsegmented section and distal segmented section are found in early osteichthyes, rhizodonts, and sarcopterygians (Johanson et al., 2005). Flexibility of these bony structures is achieved by the presence of segments in the more distal section of the fin ray. These segments are approximately the same length along the distance of the fin ray and are connected by collagen fibers (Geerlink and Videler, 1987; Videler, 1993).

During swimming, the shape of fish fins changes constantly as a result of both muscular control and hydrodynamic loading. Complex fin conformations result from movements driven by muscles at the fin ray base and also the bending of fin rays along their length. The bending of fin rays can occur due to active sliding of a single hemitrich relative to its sister hemitrich (Videler, 1993; Lauder et al., 2006; Alben et al., 2007) or passive deflection by fluid forces. In either case, the curvature of fish fins, either active or passive, has

Received 24 September 2012; Revised 29 January 2013; Accepted 8 March 2013.

Published online 30 May 2013 in

Wiley Online Library (wileyonlinelibrary.com).

DOI 10.1002/jmor.20161 
hydrodynamic implications for locomotion (Lauder et al., 2006, 2011; Mittal et al., 2006).

Previous research has investigated the anatomy, mechanics, and active control of fin rays and their flexibility during normal locomotion, and studied the mechanical properties of isolated fin rays (Lauder et al., 2006, 2011; Alben et al., 2007; Taft et al., 2008; Taft and Taft, 2012). However, there has been no detailed investigation into the flexibility of ray-finned fish fin rays under unsteady locomotor conditions such as turning, and no study to our knowledge has investigated the effect of fluid dynamic perturbations on fin ray function. Fish naturally encounter a variety of fluid jets and vortices from wakes, currents, tides, and storm surges during swimming, and a complete understanding of fin ray mechanics should include analysis of the effect of perturbations on fin ray function. Therefore, our research sought to answer two major questions: a) just how flexible are fish pectoral fin rays during normal swimming and turning, and $b$ ) what effect do fluid perturbations have on pectoral fin ray dynamics during normal swimming? We hypothesized that fish can actively stiffen their fins to have lower fin ray curvatures during turning than during swimming. We also hypothesized that flexible fin rays would yield to fluid perturbations and that perturbations would, therefore, disrupt the kinematics of the fin beat. To answer these questions, we first examined the structure of bluegill sunfish (Lepomis macrochirus) fin rays using high-resolution computed microtomography $(\mu \mathrm{CT})$ scans. Then, we investigated the threedimensional curvature of fish fin rays under normal swimming conditions (steady swimming and low-speed turning maneuvers) and with induced passive deformation by a vortex perturbation impacting the fin. Finally, we used computational fluid dynamic models to evaluate the effect of stiffness and segmentation on patterns of fin ray curvature resulting from perturbations.

\section{MATERIALS AND METHODS Fish}

Seven bluegill sunfish (Lepomis macrochirus Rafinesque, 1819 ; $6.1-18.5 \mathrm{~cm}$ total length, TL) were used in morphological $(n=6)$, swimming $(n=4)$, and vortex perturbation $(n=2)$ studies. Fish were captured from White's Pond (Concord, MA) using a seine net, kept in individual $40 \mathrm{~L}$ aquaria with a 12:12 light:dark schedule, and fed three times weekly. All fish were handled ethically according to Harvard University Institutional Animal Care and Use Committee guidelines, protocol 20-03.

\section{Morphological Analysis}

The third left pectoral fin ray was removed from six bluegill sunfish (preserved from previous experiments in the Lauder laboratory at Harvard University) ranging from 6.1 to $16.9 \mathrm{~cm}$ TL. We chose the third pectoral fin ray because it is the longest fin ray and would permit the most accurate calculation of potential maximum curvatures in the kinematic and perturbation experiments. Measurements of fin ray length and the length and number of segments in each fin ray for all fin rays were made using a Leica MZ7.5 stereo microscope (Leica Microsystems, Wetzlar, Germany) with attached Nikon Coolpix 4500 (Nikon, Melville, NY) and analyzed in ImageJ1.44p (Rasband, 1997-2012). The entire left pectoral fin and the third right fin ray from a 6.1 TL individual were sent to Cornell Imaging (Cornell University, Ithaca, NY) for high resolution $\mu \mathrm{CT}$ scanning. Samples were scanned at a voxel size resolution of 8.5 microns.

\section{Kinematics and Particle Image Velocimetry (PIV)}

Studies of fin curvature under normal steady-swimming conditions and during yaw-turning maneuvers were performed while the fish swam steadily at $0.5,1.0,1.5$, and $2.0 \mathrm{~L} \mathrm{~s}^{-1}$ in a $600 \mathrm{~L}$ recirculating flow tank with a working area of $80 \times 20 \times 20 \mathrm{~cm}^{3}$. Turns were elicited following the procedures used by Drucker and Lauder (2001b). Fish were recorded swimming in three views (lateral, XY plane; posterior, YZ plane; and ventral, XZ plane) at 250 or 500 frames per second (fps) using three synchronized Photron Fastcams (Photron USA, San Diego, CA) each with a resolution of $1024 \times 1024$ pixels. Studies of fish hovering were performed while the fish maintained a steady position in still water in a $10 \mathrm{~L}$ aquarium $(29 \times 18 \times 14$ $\mathrm{cm}^{3}$ ) and filmed in three views at $500 \mathrm{fps}$. The three video planes were calibrated in three dimensions using direct linear transformation of a custom 40-point calibration object and digitized using DLTdv5, a program written for MATLAB (R2011a, MathWorks, Natick, MA) by Hedrick (2008). Three swimming sequences were selected for careful highly detailed analysis of steady swimming and weak and strong side turning maneuvers. For each of the three sequences, we digitized the positions of every fin ray in 20 video frames spaced equally through time. About 200 points were digitized for each video frame or about 4000 points per sequence. The density of points corresponds to one point every $2-3 \mathrm{~mm}$ along each fin ray, where rays branched, points were digitized along the most dorsal segment.

Particle image velocimetry (PIV) was performed using $50 \mu \mathrm{m}$ diameter particles illuminated by a single beam of a $10 \mathrm{~W}$ continuous-wave argon-ion laser (Innova 300 Series, Coherent Laser group, Moorpark, CA) focused into a 1-2 mm thick horizontal light sheet using a custom built optic and mirror system as in our previous research (Drucker and Lauder, 2001b; Lauder and Drucker, 2004; Lauder et al., 2006). The horizontal light sheet was set at same height as the center of the vortex ring generator and recorded in the ventral (XZ) plane at $1000 \mathrm{fps}$.

\section{Perturbation}

A custom vortex generator was designed to perturb the fish during normal hovering behavior following the methods outlined in Tangorra et al. (2011). Food dye injected into the vortex cavity before it was expelled permitted visualization of the vortex in the high-speed videos. Fish were placed in $10 \mathrm{~L}$ tank with vortex generator mounted to an inside wall, and left undisturbed until they assumed a normal hovering behavior in front of the vortex generator (Fig. 1). Vortex perturbations included impacts with both the pectoral fin and body of the fish during normal hovering. Perturbation experiments were recorded at $500-1000 \mathrm{fps}$ for later analysis of vortex velocity, fin deformation and curvature, and fish kinematics. Vortex perturbations traveled at a velocity of $0.97 \pm 0.007 \mathrm{~ms}^{-1}$ and impacted the fin with an estimated force of $67 \mathrm{mN}(2.3 \mathrm{mN}$ s.e., Tangorra et al., 2011). The force estimated for the vortex generator was calculated by aiming the vortex at a flat aluminum plate at distances of 10 and $15 \mathrm{~cm}$ from the generator orifice (the distance at which the fish pectoral fin was typically located). The plate was attached to a $250 \mathrm{~g}$ force transducer (model LSB200, JR S-Beam load cell, Irvine, CA). Signals from 


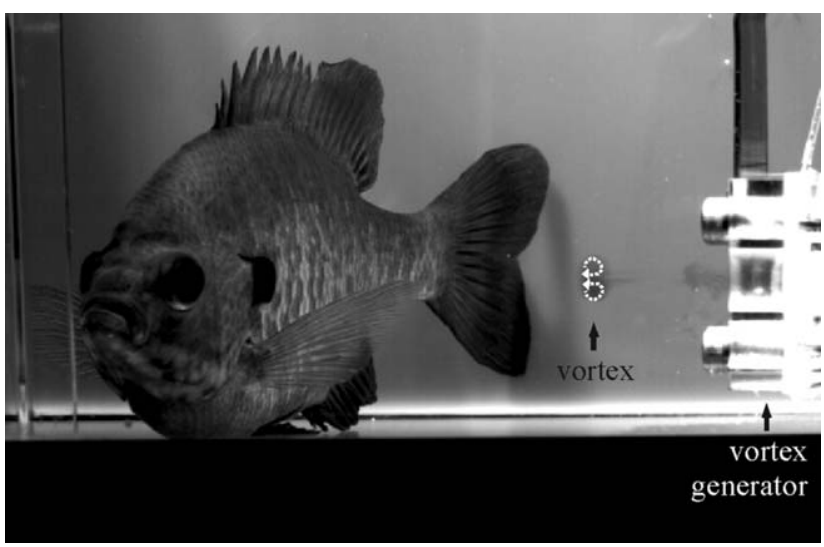

Fig. 1. Bluegill sunfish performing hovering swimming behavior in front of custom built vortex generator. Vortex approaching the fish was traveling at approximately $1 \mathrm{~ms}^{-1}$ and is filled with dye, but has been highlighted in this figure with dashed white curved arrows to enhance visibility.

the force transducer were converted to digital using a PCI-6221 (LabView8, National Instruments, Austin, TX) and recorded using a customized LabView program to convert volt signals into force data.

Flexural stiffness (or bending rigidity or stiffness, i.e., the force couple required to bend a nonrigid structure to a unit curvature) of the fin ray was measured by calculating the deflection of the fin ray as a result of vortex perturbation using the equation (Eq. 1):

$$
\frac{F L^{3}}{3 y}
$$

where $F$ is the force of the perturbation, $L$ is the length of the fin ray, and $y$ is the distance the ray was deflected.

\section{Models}

We modeled and simulated the interactions of fin rays with vortex dipoles in a $2 \mathrm{D}$ fluid. The model was previously used to study the swimming of flexible foils and body-vortex interactions at high Reynolds number (Alben, 2009, 2012; Alben et al., 2012). We refer to these works for technical background on the model. We modeled a bending fin ray as a passive flexible beam in a $2 \mathrm{D}$ fluid. We assumed that the fin ray is connected to a body at one end, and rotates freely there (a pinned or hinged boundary). At the other end, the fin ray had a free-end boundary condition. The coordinate system used for the model was adapted to fit the context of the live fish studied (e.g., XY plane in the lateral view of the fish, $\mathrm{XZ}$ plane in the ventral view of the fish, and YZ plane in the posterior view of the fish). The fish body was modeled as an infinite straight wall along the $x$ axis, which fluid does not penetrate, and to which the fin ray was connected at the origin. Due to its infinite extent, the wall is a somewhat stronger barrier than is the fish body, but its main role is to provide a realistic resemblance to the geometry where the fin ray attaches to the body. In each of our simulations, a vortex dipole was initialized at a finite distance from the fin ray. Subsequent to the initial time, the motion of the dipole and the fin ray was found by solving the equations of 2D fluid dynamics (the incompressible Euler equations with slip boundary conditions on the fin ray and wall) together with the equation for the dynamics of a bending beam (Alben, 2009). Each vortex in the dipole pair is a smoothed blob, with a parameter $d=0.1$, giving the typical spread radius, and corresponding to the vortex rings' radii in our experiments. The functional form of the blob is that given by Krasny (1986).
The key dimensionless parameter governing the interaction is (Eq. 2):

$$
B=\frac{\mathrm{EI}}{\rho \Gamma^{2} L W},
$$

where EI is the bending modulus, $\rho$ is the fluid density, $\Gamma$ is the vortex ring circulation, $L$ is the fin ray length, and $W$ is the spacing between fin rays in the plane of the fin. The range of flexural rigidity (EI) estimates along the length of the fin ray produced for the model was taken from previous work calculating the flexural stiffness of bluegill sunfish pectoral fin rays (Lauder et al., 2011). In this work, a typical value of EI for the basal $38 \%$ (unsegmented) portion of the fin ray was $3.2 \mathrm{~N}$ $\mathrm{m}^{2} \times 10^{-6}, \rho$ was $1000 \mathrm{~kg} \mathrm{~m}^{-3}, \Gamma$ was $0.02 \mathrm{~m}^{2} \mathrm{~s}^{-1}, L$ was 0.04 $\mathrm{m}$, and $W$ was $0.001 \mathrm{~m}$. With these parameters, $B$ was 0.2 in the basal region. CT measurements indicated a rapid (exponential) decay of the moment area of inertia with distance from the fin ray base, in the segmented region. Therefore, we set $B(s)(s$ is arc length normalized by total fin ray length) to: 0.2 for $0 \leq s \leq 0.38,0.2 \times \exp (-|s-0.38| / \delta)$ for $0.38 \leq s \leq 1$. We also emulated the presence of discrete segments by using an alternative $B(s)$ in the segmented region $0.38<s<1$, given by $0.2 \times \exp (-|s-0.38| / \delta) \times|\sin (k s)|$, with $k$ values of 15 (for three segments), 30 (for six segments), or 60 (for 12 segments). Here, $\delta$ is the exponential decay rate, which was varied from 0.04 (rapid decay of $B$ ) to 3 (slow decay; nearly uniform $B$ ) in our simulations. According to Lauder et al. (2011), EI decays by about a factor of 10 from $s=0.38$ to $s=0.67$, giving $\delta=0.13$ for biological specimens.

\section{RESULTS}

\section{Fin Ray Morphology}

A single fish fin ray, or lepidotrich, is composed of two halves (the medial and lateral hemitrichs, in the case of the pectoral fin). The longest pectoral fin ray, third from the dorsal edge (Fig. 2A, B), is unsegmented for the proximal $40 \%$ of its length. The distal $60 \%$ of the fin ray is comprised of serially stacked segments. Comparison of fin rays of individuals between 6.1 and $16.9 \mathrm{~cm}$ TL demonstrated that while the number of segments increases with ontogeny (Fig. 2C), the relative proportion of the segmented to solid portion of the fin ray $(60: 40)$ remains relatively constant in bluegill, as does the area of separated branching (distal $20 \%$ of fin ray). Within a single fin, the same unsegmented proportion of the total fin ray length remains constant among fin rays 2 (dorsal-most) to $11(39.4 \pm 2.3 \%$ total fin ray length, mean \pm s.d.), and approaches 50\% unsegmented portion in the three ventral-most fin rays. The length of segments was also conserved throughout the fin in a single individual, but comparisons among individuals suggest a pattern of negatively allometric growth of fin ray segments with respect to fish total length through ontogeny $(3.5 \pm 0.3 \%$ total fin ray length, $6.1 \mathrm{~cm}$ TL individual; $1.2 \pm 0.01 \%$ total fin ray length, $16.9 \mathrm{~cm}$ TL individual). The segments of the fin ray do not abut as flat ends; instead each segment is tapered at its proximal and distal ends such that the angle between 

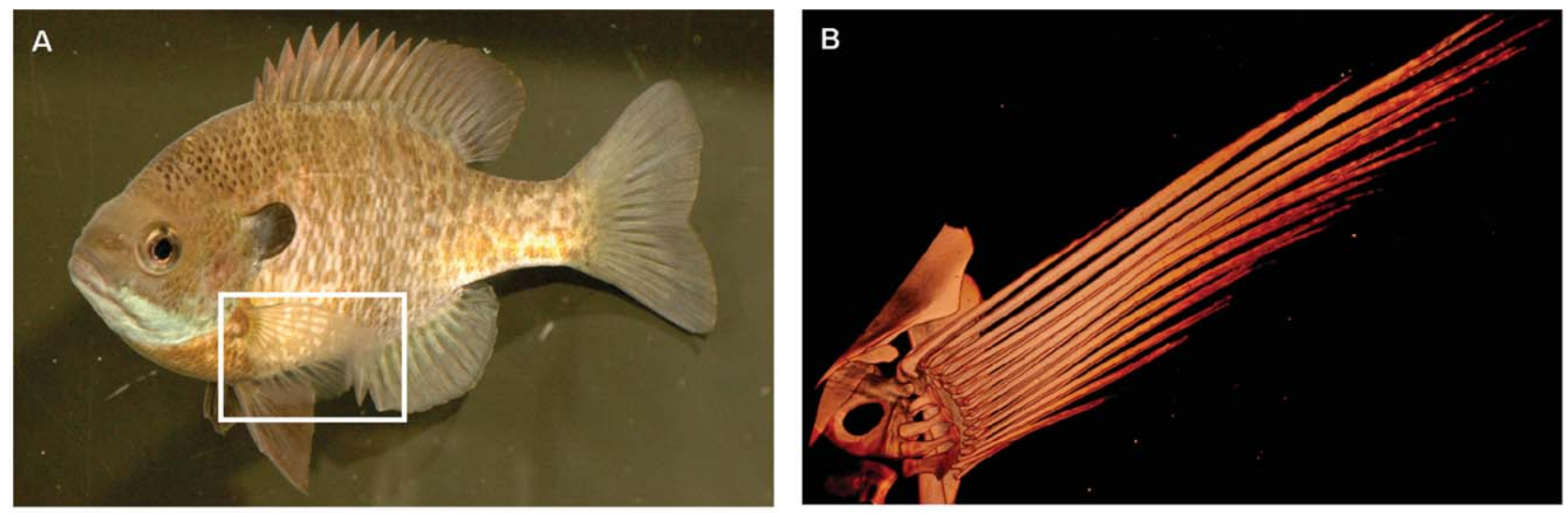

C
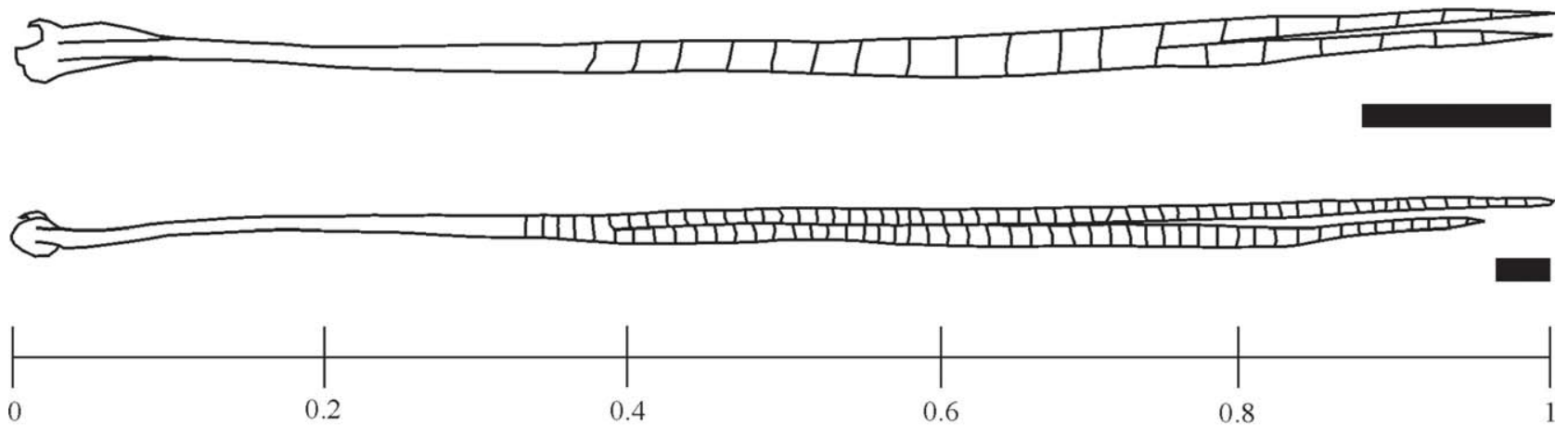

proportion of fin ray length

D
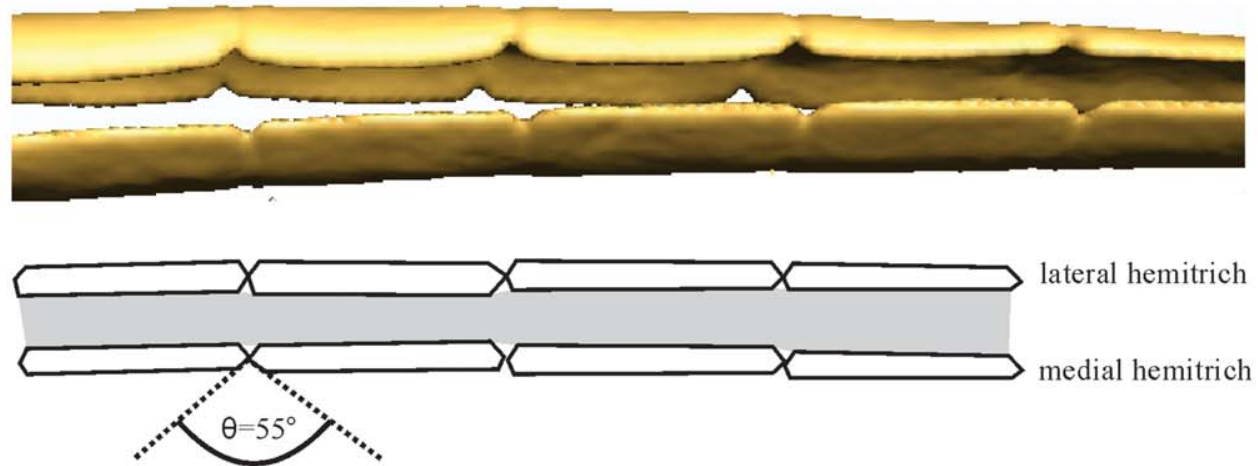

Fig. 2. Fin ray structure. A: Hovering bluegill sunfish (with mirror reflection also visible), inset denotes pectoral fin shown in panel B. B: Computed microtomography $(\mu \mathrm{CT})$ scan of left pectoral fin and girdle of $6.1 \mathrm{~cm}$ TL sunfish (voxel size $=3.7 \mu \mathrm{m}$ ). C: Tracing of longest (3rd) left pectoral fin ray, from a $6.1 \mathrm{~cm}$ TL sunfish (top) and a ray from a sunfish of $16.9 \mathrm{~cm}$ TL (bottom, on which the vortex perturbation experiments were performed). Scale bars are $1 \mathrm{~mm}$. D: Zoomed-in view of segments from the $\mu \mathrm{CT}$ scan in B (top) and tracing of longitudinal section through third fin ray of the $16.9 \mathrm{~cm}$ TL specimen (bottom), showing four serial segments in ventral view. Gray shading represents collagen and elastic fibers connecting lateral and medial hemitrichs.

segments is approximately $55^{\circ}$ (Fig. $2 \mathrm{D} ; 55.6 \pm$ $1.7^{\circ}, n=6$ fin rays).

\section{Normal Swimming and Turning Maneuvers}

Range of motion of individual pectoral fin rays. During steady swimming, pectoral fin abduction was initiated by the dorsal-most (ray 1) and ventral-most (ray 14) fin rays pulling away from the body; sequential abduction of individual fin rays continued until the rays in the center of the fin (rays 7 and 8) were the last to leave the side of the fish. The paths of ray 1 and 14 during abduction and adduction were nearly horizontal (red and blue, Fig. 3A). Ray 7 was curved upwards during most of abduction but straighter during the adduction, moving in a conical shape in a counterclockwise direction (green, Fig. 3A). The medial ray 7 began the fin beat by rising and staying close 

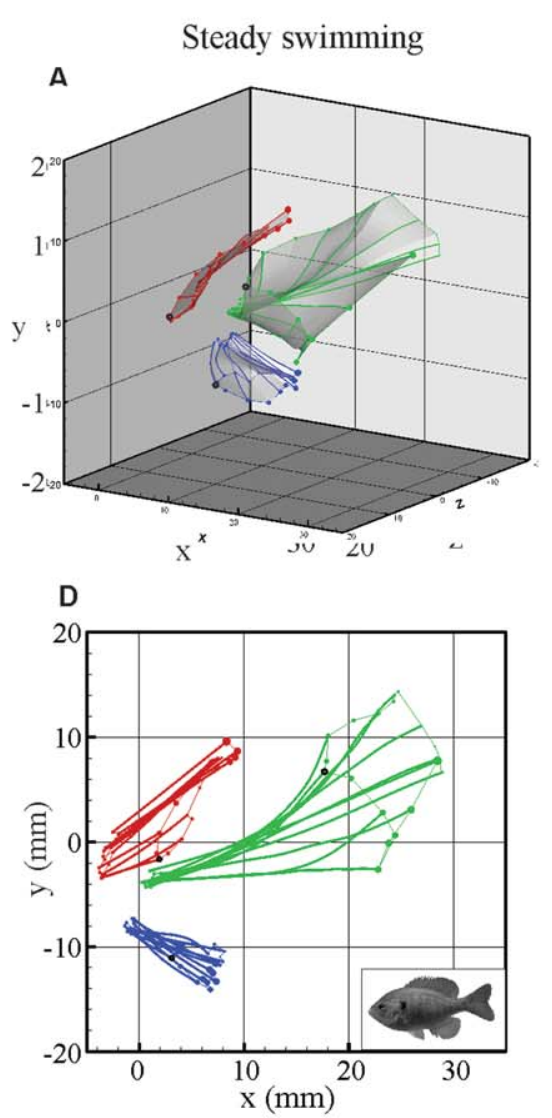

G

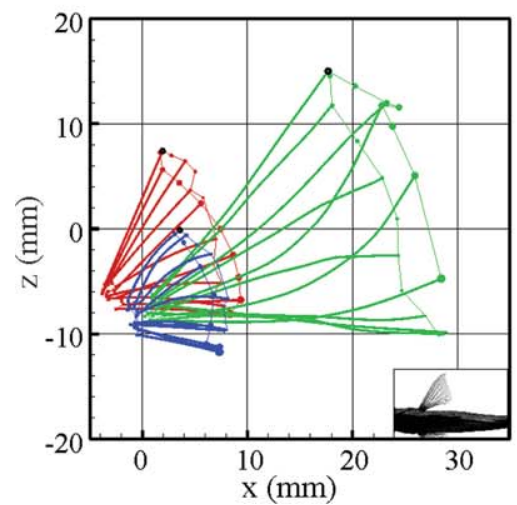

Turn,

weak side

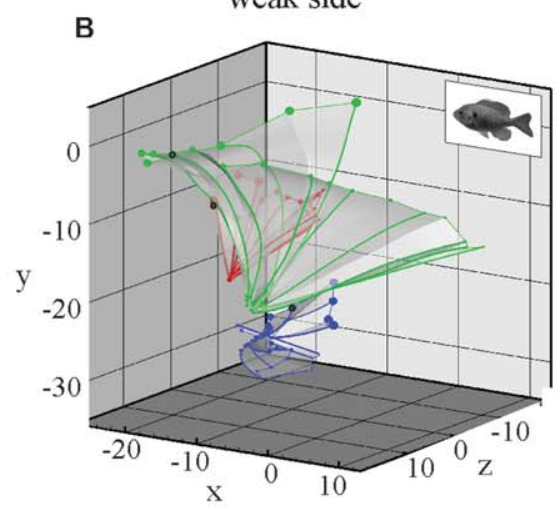

E

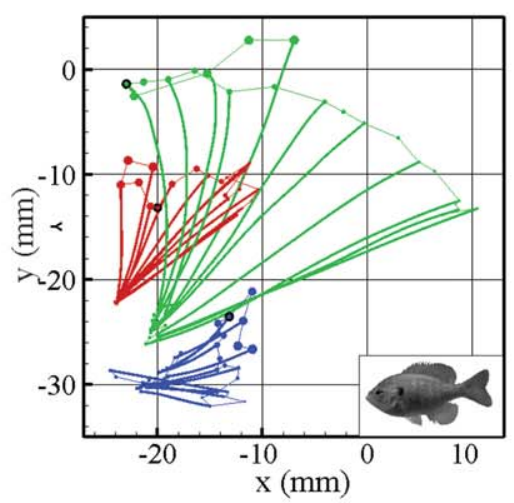

H

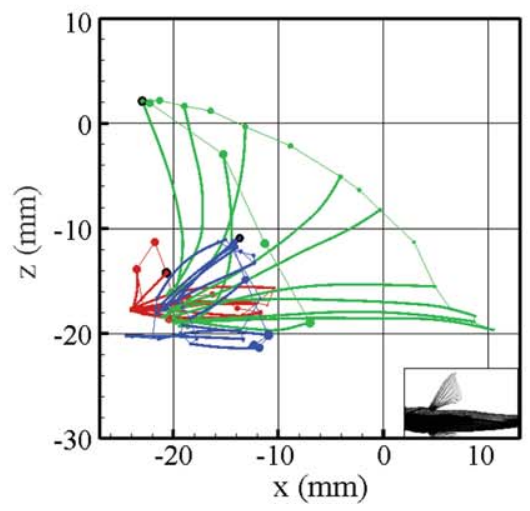

Turn,

strong side

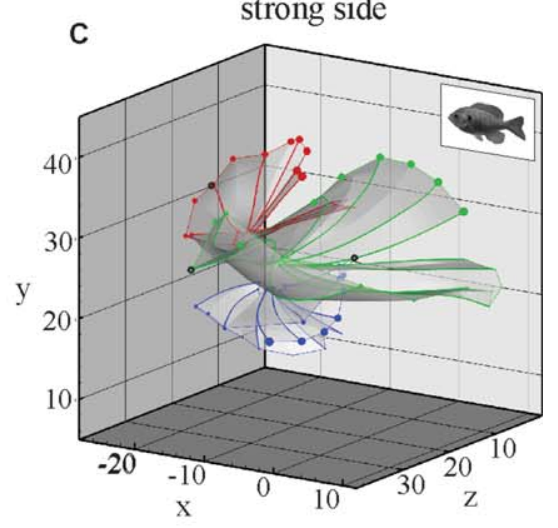

$\mathrm{F}$

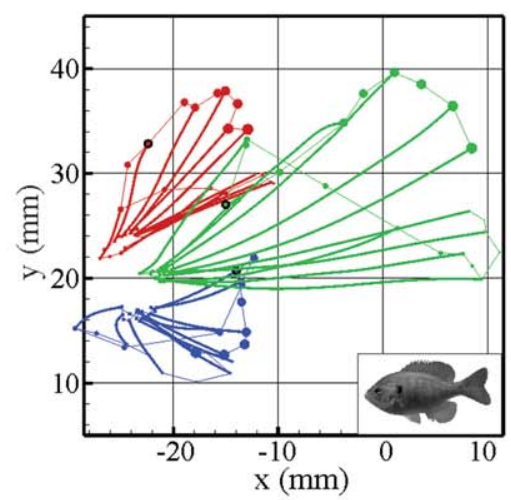

I

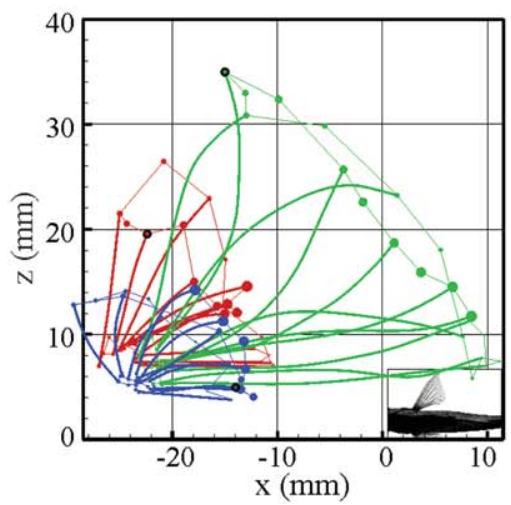

Fig. 3. Paths of individual fin rays through time for steady swimming $\left(1.0 \mathrm{Ls}^{-1}\right.$, first column), a weak side maneuver $\left(0.5 \mathrm{Ls}^{-1}\right.$, second column), where the fish turns away from the stimulus, and a strong side maneuver $\left(0.5 \mathrm{Ls}^{-1}\right.$, third column), where the fish turns toward the stimulus. Paths of the dorsal ray (ray 1), middle ray (ray 7), and ventral ray (ray 14) are shown in red, green, and blue, respectively. A-C: Three-dimensional views. D-F: Lateral views. G-I: Ventral views. Inset images of a sunfish show the plot orientation. Circle size at the ray tips increases with time to show the direction of motion (from abduction through adduction). Point circled in black at tip of fin ray at transition point from abduction to adduction. Comparisons of the lateral views (D-F) and ventral views $(\mathrm{G}-\mathrm{I})$ show the difference in angles of excursion of the different rays.

to the body before sweeping out and dropping down toward the horizontal plane. In the lateral view (Fig. 3D), there was considerable upwards curvature of ray 7 through the early to middle times of the beat. In the ventral view (Fig. 3G), ray 7 curved in toward the body during the outstroke and away from the body during the instroke.
During a turning maneuver, fin rays on the weak side (the side away from the stimulus) had larger angular excursions in the lateral (Fig. 3E) and ventral view (Fig. $3 \mathrm{H}$ ) as compared to steady swimming. In the lateral view, rays 1 and 7 were near vertical at their most forward positions, and in the ventral view, rays 1 and 7 swept out to 
about $90^{\circ}$ from the body. Unlike steady swimming, weak side fin rays finished their fin beat dorsal to their starting point. Fin ray 7 curved into the flow during abduction into the flow on the weak side fin beat (green, Fig. 3E). The tip of the dorsal ray (ray 1 , red, Fig. $3 \mathrm{H}$ ) was never far from the body, even as it angled upwards (lateral view, Fig. 3E).

Similar to the weak side fin, the rays of the strong side fin followed mostly clockwise paths (Fig. 3C) that finished dorsal to their start positions. However, while the range of angular motion of the dorsal ray (red, Fig. 3F, I) was similar to the range of motion in steady swimming, the abduction and adduction paths followed were very different. Backwards curvature of ray 7 during abduction and outward curvature during adduction were consistent with passive deflection of the ray by the fluid dynamic forces (green, Fig. 3I). The ventral ray had underwent its greatest range of motion during the strong side maneuver; ray 14 moved well forward of the base during the abduction phase (blue, Fig. 3F, I) and returned to the body dorsal of its start position.

Curvature of the whole pectoral fin. During steady swimming (Fig. 4A, D), spanwise curvature of the pectoral fin was mostly negative (curved away from the flow) during abduction $(0-0.25 \mathrm{~s})$ and positive (curved into the flow) during adduction (0.25-0.45 s), and did not exceed $\pm 0.05 \mathrm{~mm}^{-1}$. In contrast, during a weak side maneuver, the spanwise curvature was close to zero or positive during much of the abduction or holding phase $(0-0.8 \mathrm{~s})$ as the fin rays were curved forward into the flow (Fig. 4B,E). The upper distal region of the fin (blue line, Fig. 4B), where the fin rays are more flexible and less able to resist fluid forces, had an average negative curvature during the holding phase. During the strong side maneuver, the curvature was negative during abduction and positive during adduction, similar to the curvature during a steady beat (Fig. 4C,F).

Chordwise curvature was positive when the fin region was cupped into the flow and negative when cupped away from the flow during steady swimming; magnitudes of the chordwise curvature were considerably larger than the spanwise curvature, reaching a maximum of $\pm 0.5 \mathrm{~mm}^{-1}$ (Fig. $4 \mathrm{G}, \mathrm{J})$. In the dorsal portion of the fin, the very flexible fin rays in the distal region (blue) bent easily in the flow and had the highest curvatures. During most of adduction, the curvature in the base and medial regions were very close to zero (from 0.3 to $0.45 \mathrm{~s}$; Fig. 4J). Weak side fin chordwise curvature was most notable in the distal (blue) region of the fin as well (Fig. 4H). Chordwise curvature was minimal in the ventral portion of the fin on both weak and strong side fins (Fig. 4K,L).

\section{Fin Curvature During Hovering}

Bluegill sunfish routinely hovered under no flow conditions and in the absence of a provocative stimulus. Hovering behavior was characterized by an alternating (left-right-left) pectoral fin cadence, during which time other fins were largely inactive and only exhibited minor corrective movements. During a single hovering fin beat, the left pectoral fin (Fig. 5A) was abducted in a nearly flat fin conformation; adduction was led by cupping of the dorsal aspect of the pectoral fin as it returned alongside the body. During the hover beat, the longest pectoral fin ray (third from the dorsal edge) showed a curvature pattern of two waves being passed along its length: During abduction there was a wave from base to tip, and during adduction there was a wave from tip to base (Fig. 5B,C). However, curvature of the third fin ray was very low (Fig. $5 \mathrm{D}, \mathrm{E}$ ); the maximum curvature of the fin ray during normal hovering was $0.029 \mathrm{~mm}^{-1}$ in the proximal, unsegmented portion of the fin ray and $0.054 \mathrm{~mm}^{-1}$ in the distal, segmented portion of the fin ray.

\section{Vortex Perturbation of Fins}

Vortex impact on the pectoral fin during hovering resulted in deformation of the shape of the fin (Fig. 6A) but no change in the adduction pattern of the fin from normal behavior (Fig. 6B). Relative curvature mapped over time (Fig. 6C) showed that vortex impact on the fin ray did not result in a smooth bending of the fin. Instead, the fin ray deformation consisted of small, localized areas of bending, with curvatures greater than $5 \mathrm{~mm}^{-1}$ (illustrated by the small areas of high counter-curvature and hence alternating color in Fig. 6C). Maximum curvature observed at time of vortex impact was $9.38 \mathrm{~mm}^{-1}$, which was significantly higher than the curvatures observed under any of the unperturbed swimming conditions $(P<0.0001$; one-sample $T$-test calculated by hand). Flexural stiffness of the fin ray was calculated to be $565 \times 10^{-6} \mathrm{~N} \mathrm{~m}^{2}$.

PIV analysis of vortex impact on the pectoral fin confirmed that deformation in the shape of the fin and increased curvature of the fin ray was a result of impact of the vortex with the fin ray (Fig. 7A). Vortex impact caused the fin ray to bend in compliance from the force of the moving fluid. Curvature of the third fin ray appears to be relatively uniform at large scale (Fig. 7B), but investigation of relative curvature over time at small regions within the fin ray showed regions of localized bending or buckling (curvatures greater than 5 $\mathrm{mm}^{-1}$ ) at the point of vortex impact (Fig. 7C,D).

\section{Models of Vortex Perturbations}

Effect of stiffness. We simulated impacts of vortex dipoles with fin rays with various distributions of bending stiffness. Figure 8A shows five 

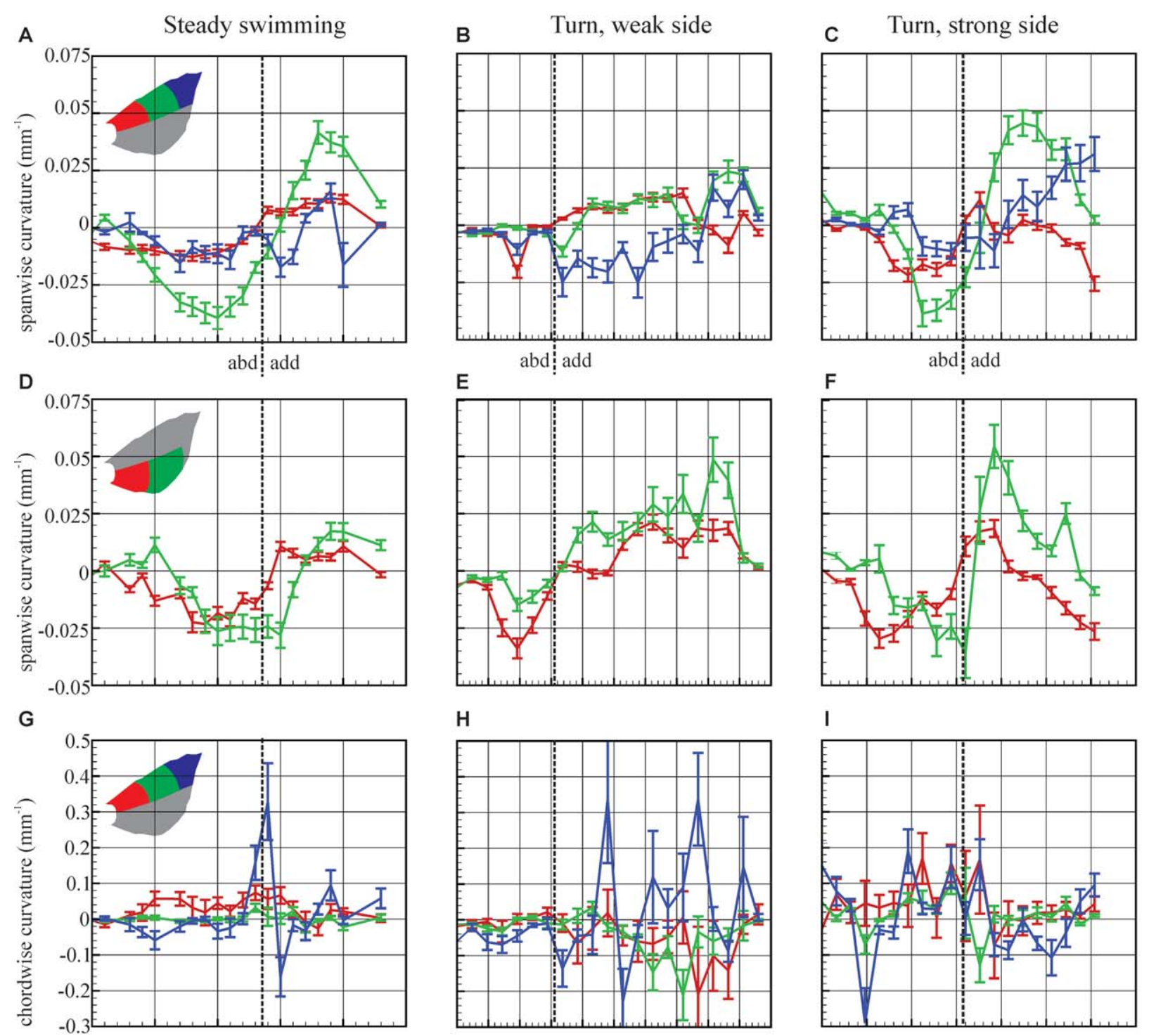

$\mathrm{H}$
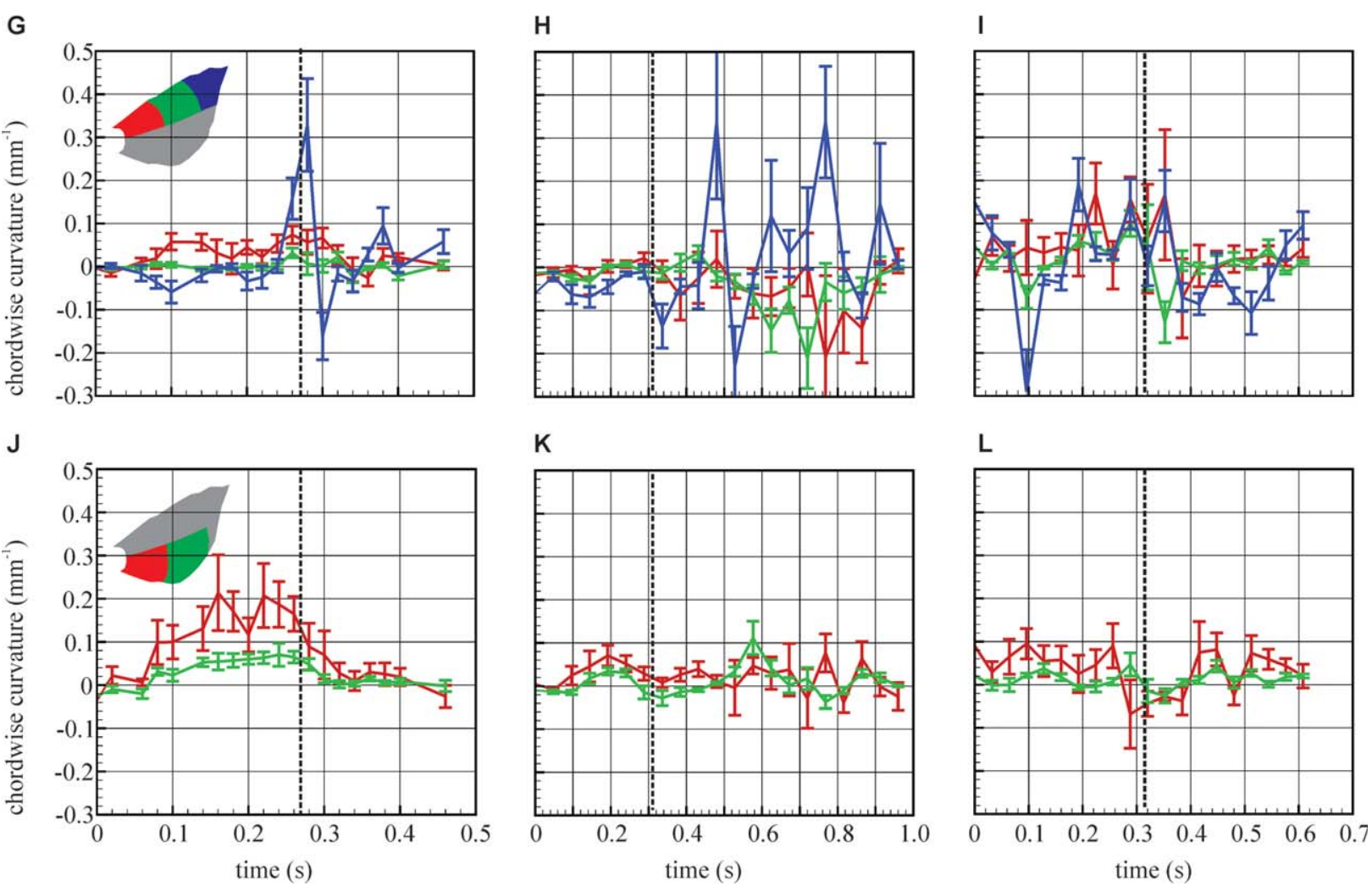

Fig. 4. Spanwise and chordwise curvature of the pectoral fin during steady swimming (first column), a weak side maneuver (second column), and a strong side maneuver (third column). Fin icons in the first column show the region of the fin averaged for that row of plots. A-C: Spanwise curvature of the fin dorsal half. The vertical dashed line represents the transition between abduction (abd) and adduction (add) for all panels below. D-F: Spanwise curvature of the fin ventral half. G-I: Chordwise curvature of the fin dorsal half. J-L: Chordwise curvature of the fin ventral half. The base, medial, and distal regions of the fin are plotted in red, green, and blue, respectively. Positive curvature indicated that this region of the fin is cupped forward into the flow, while negative curvature shows the fin to be cupped backwards away from incident flow. Data points represent the mean $(n=4) \pm 1$ SD. 
A
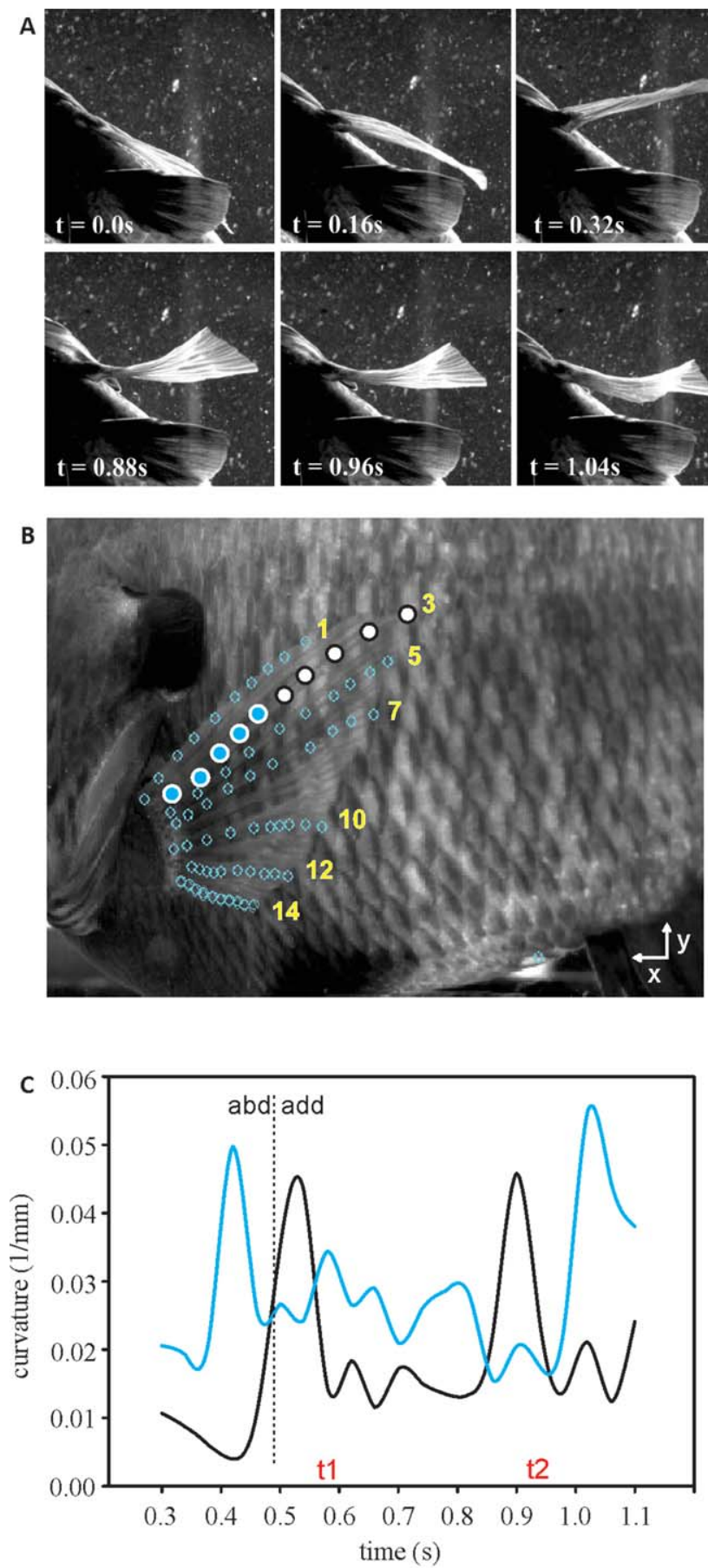
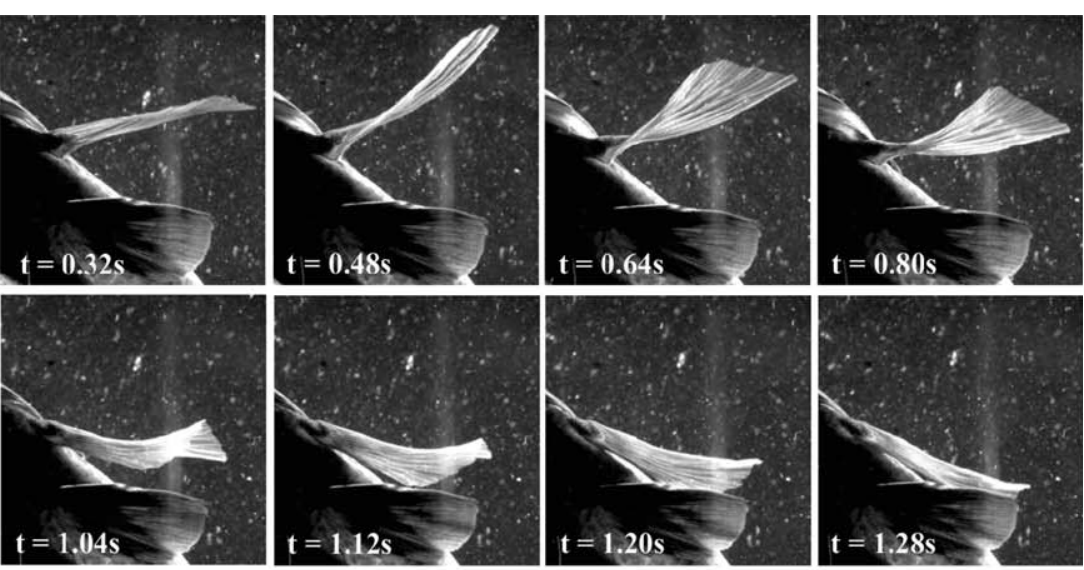

D

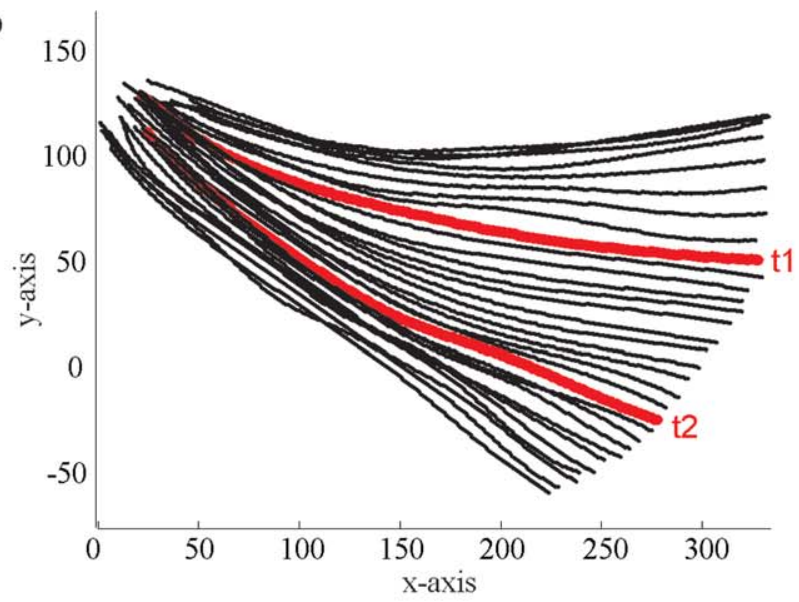

$\mathbf{E}$

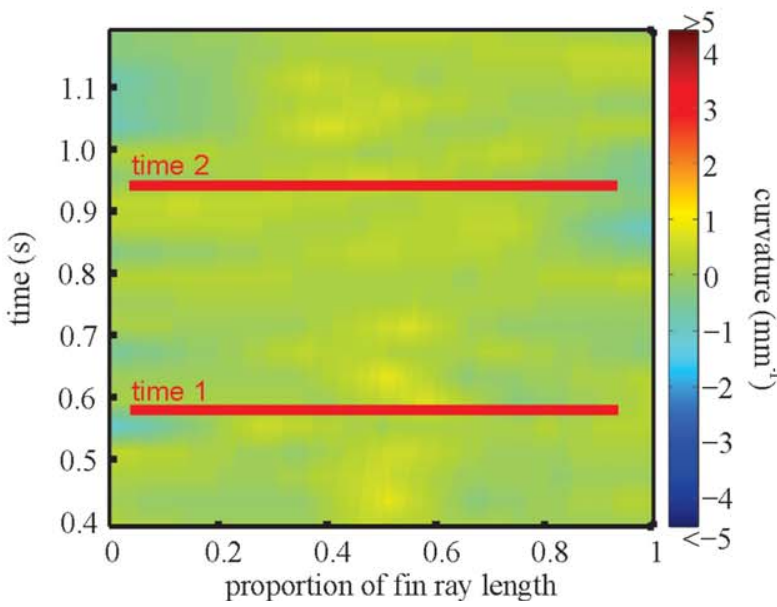

Fig. 5. A: Video sequence of a pectoral fin beat during normal hovering behavior. B: Analysis of the fish pectoral fin. Ten points were selected along fin rays (from dorsal to ventral) 1, 3, 5, 7, 10, 12, and 14 to produce spline curves describing fin bending during hover. The curvature of the base of the fin (proximal 5 points, blue circles with white border) and tip of the fin (distal 5 points, white circles with black border) are compared in $\mathbf{C}$. C: Curvature of the proximal (blue) and distal (black) portions of the longest pectoral fin ray (third from dorsal edge) during a hovering beat. Transition between abduction (abd) and adduction (add) is marked by a vertical dashed line. D: Position of longest (3rd from dorsal edge) fin ray from time 0.48 to $1.20 \mathrm{~s}$ during adduction of the pectoral fin. E: Curvature of the longest fin ray over time. Fin ray position ( $x$-axis) is from base ( 0 , proximal) to tip (1, distal) of the fin ray. The color bar indicates degree and relative direction of curvature (in units of $1 / \mathrm{mm}$ ). Curvature is relative and standardized to a maximum of five for comparison among behaviors (see text for further discussion). Data presented here are from a single fin beat.

panels of fin ray and dipole snapshots (distinct moments in time), for five different values of bending stiffness decay rate $\delta$. In each panel, the tracks of the dipole pair through time are shown as straight lines connecting asterisks (the dipole vortices). 

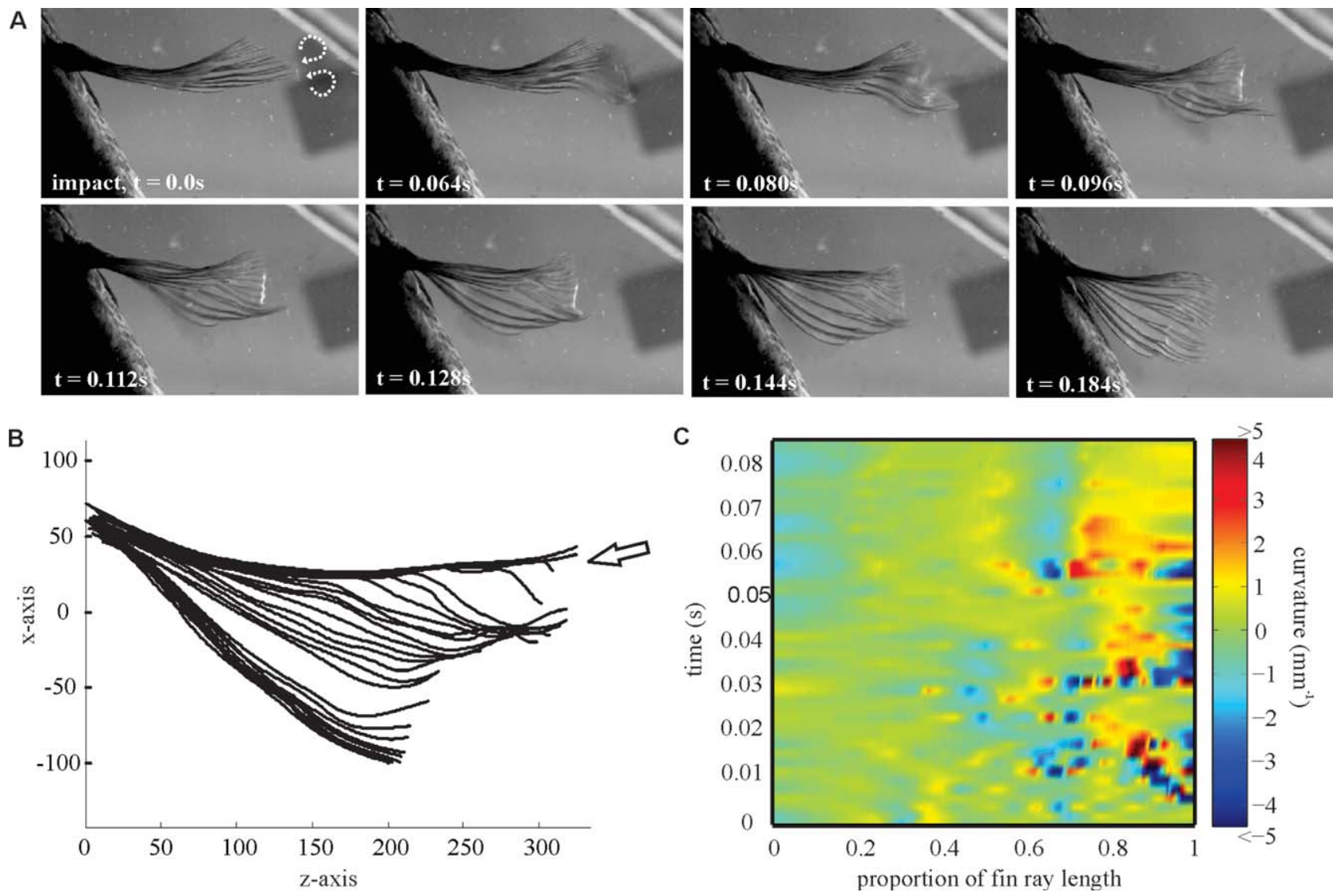

Fig. 6. A: Video sequence of vortex impact on the left pectoral fin $(t=0.0 \mathrm{~s})$ during normal hovering behavior. Position of the vortex is indicated by dashed white curved arrows. Kinematic position of the fin at time of vortex impact is the same as time $=0.8 \mathrm{~s}$ in Figure 5A. Video was recorded at $500 \mathrm{fps}$. B: Position of longest (3rd from dorsal edge) fin ray from two frames before impact through the video sequence shown in A. The arrow illustrates the direction of vortex impact. C: Curvature of the longest fin ray over time. Fin ray position $(x$-axis $)$ is from base $(0$, proximal) to tip (1, distal) of the fin ray. The color bar indicates degree and relative direction of curvature $( \pm 1 / \mathrm{mm})$. Curvature is relative and standardized to a maximum of five for comparison among incidents. Data presented here are from a single perturbation.

At the initial instant, the fin ray extends perpendicularly (horizontally) from the wall. A vortex dipole (a pair of equal and opposite point vortices of unit strength) is initially placed at one fin ray length away from the point on the fin ray $80 \%$ distal from the base. The distance between the dipole members is initially $15 \%$ of the fin ray length. These values are intended to approximate the conditions of one of the experiments (Fig. 7). The dipole is initialized at the upper right of the panel, oriented in a direction $-120^{\circ}$ from horizontal, so that initially it travels downward and to the left, towards the fin ray.

The dipole vortices diverge as they approach the fin ray. Meanwhile, the fin ray is repelled by the fluid jet between the dipole, and rotates away from it. However, the more distal, more flexible end of the fin ray is attracted to the more distal member of the dipole pair, and curls around it. A vortex sheet is continually shed from the free end of the fin ray and is shown at the final instant only as a thin gray line. The vortex sheet wraps around the distal dipole pair member (now a free vortex), while the other dipole member translates upward along the body plane. The five separate panels of Figure 8A show that when the fin ray is more flexible (left panels), it is more strongly attracted to the distal vortex. The attraction of the fin ray to the distal vortex is also seen in the experiment (Fig. 7), and may indicate a typical interaction between a flexible body and a vortex (Alben, 2012).

Figure 8B shows color maps of fin ray curvature vs. time corresponding to the snapshots in Figure 8A. The most flexible fin ray model experienced very high curvatures concentrated in small areas near the distal tip of the ray (Fig. 8A,B, far left). Increasing the stiffness of the fin ray resulted in lower curvature maximum values $(P<0.001 ; T$ test against known mean calculated by hand), but more of the fin ray experienced curvature values near the maximum. In the two stiffest models, the entire fin ray exhibited rapidly oscillating curvatures as a result of vortex impact (Fig. 8A,B, 
A

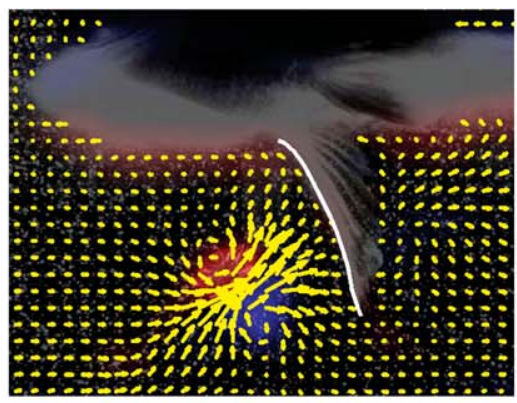

B

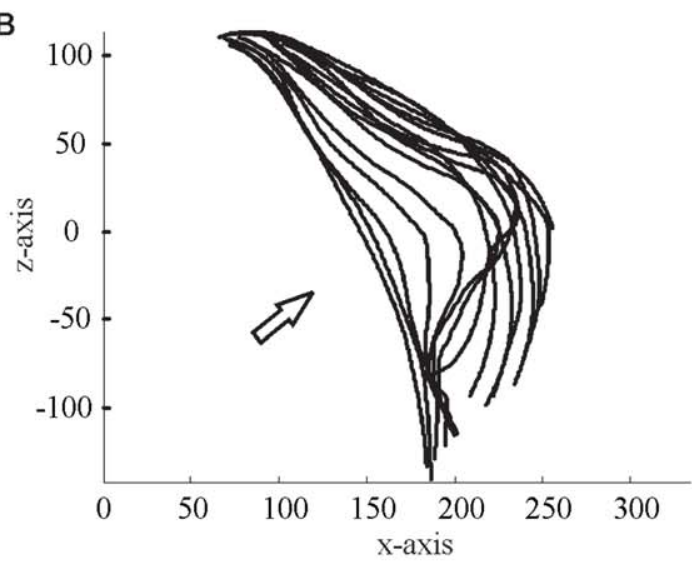

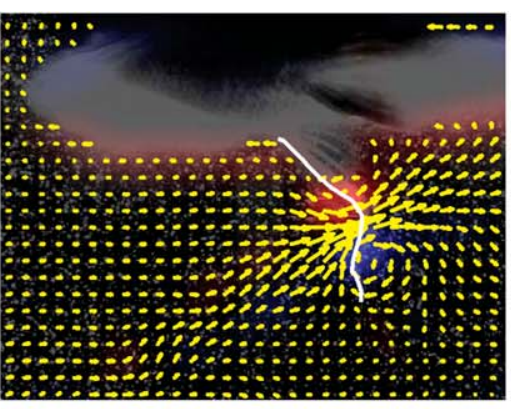

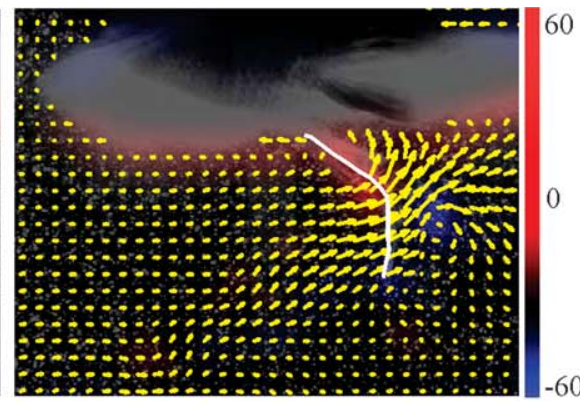

C

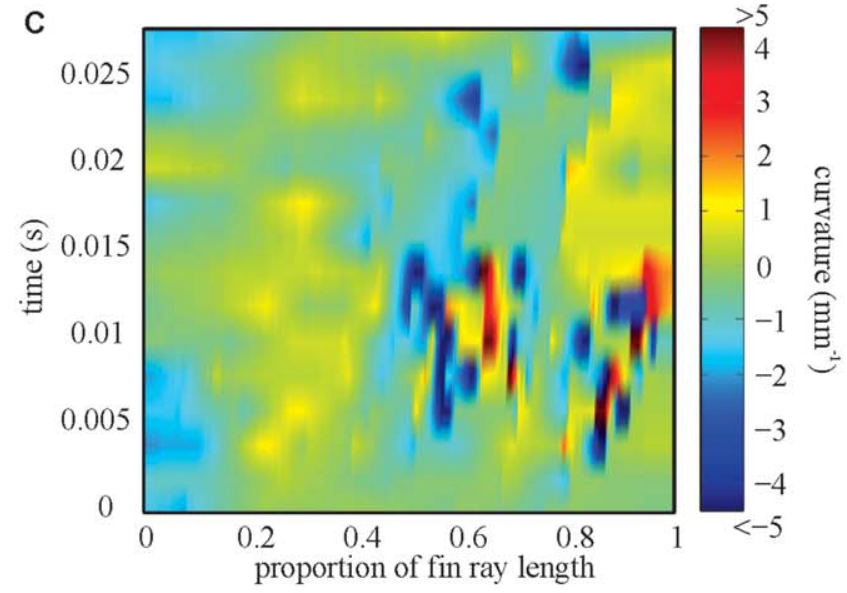

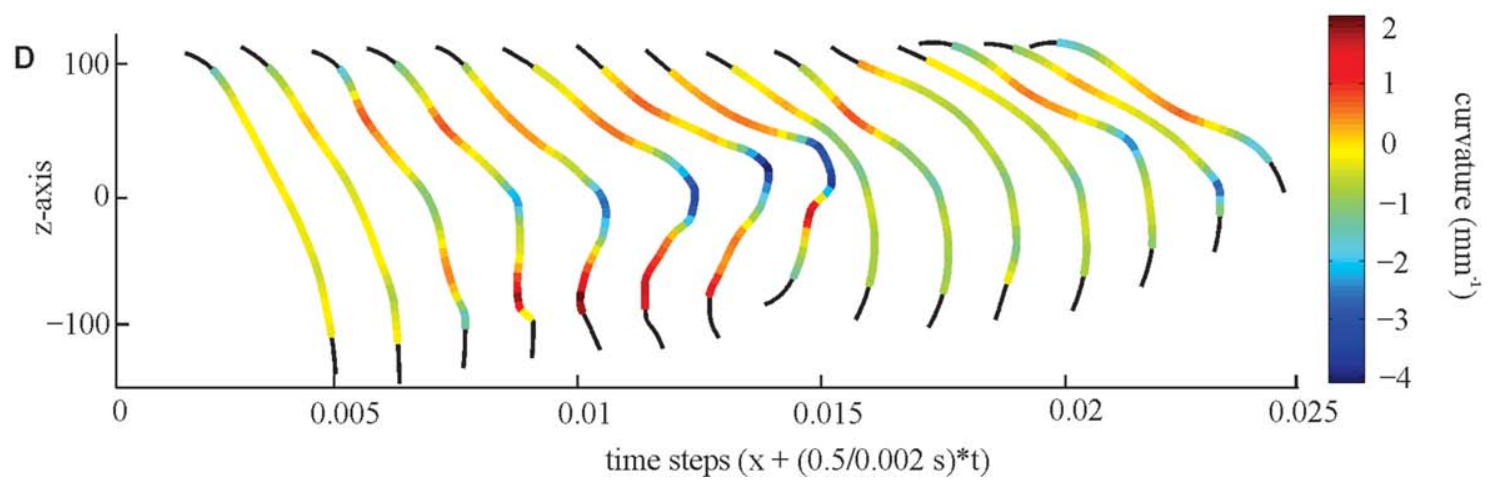

Fig. 7. A: Particle image velocimetry of vortex impact on the right pectoral fin during normal hovering behavior. Video was recorded at $500 \mathrm{fps}$. The white line has been added to highlight the fin ray of interest (the longest) in this sequence. B: Position of longest (3rd from dorsal edge) fin ray from two frames before impact through the video sequence shown in A. The arrow illustrates the direction of vortex impact. C: Curvature of the longest fin ray over time. Fin ray position (horizontal axis) is from base (0, proximal) to tip (1, distal) of the fin ray. The color bar indicates degree and relative direction of curvature $( \pm 1 / \mathrm{mm})$. Curvature is relative and standardized to a maximum of five for comparison among incidents. D: Time series of curvature of the longest fin ray over time. The color bar indicates absolute curvature along the fin ray, which was smoothed to fit the time series captured. Note that C and D have different scales. Data presented here are from a single perturbation.

right). When the vortex dipole impacted the stiffer fin ray, it was deflected at a more acute angle and rotated back into the path of the fin.

For a range of bending stiffness distribution (Fig. 9A), we computed the maximum local curvature over the fin ray during impact, and the maximum magnitude of the spatially-integrated force on the fin ray (Fig. 9B). The values are plotted vs. $B_{\text {distal }}$, the value of $B$ at a location $67 \%$ of the ray length from the base. Over an intermediate range of $B_{\text {distal }}$ (which includes a biological value, boxed), the maximum curvature is nearly inversely proportional to $B_{\text {distal }}$. Surprisingly, the maximum force decreases as the fin ray becomes more flexible. Although the distal region bends more sharply when it is more flexible, it cannot resist large fluid forces due to its small bending rigidity. Thus, the fluid force on the fin ray near the vortex is constrained to be small.

For a very flexible fin ray $(\delta=0.048)$, we found rapid oscillations of curvature near the distal $20 \%$ of the fin ray during the impact (Fig. 9C). The alternating patches of blue and red show the large values of oppositely signed curvature. Decreasing 

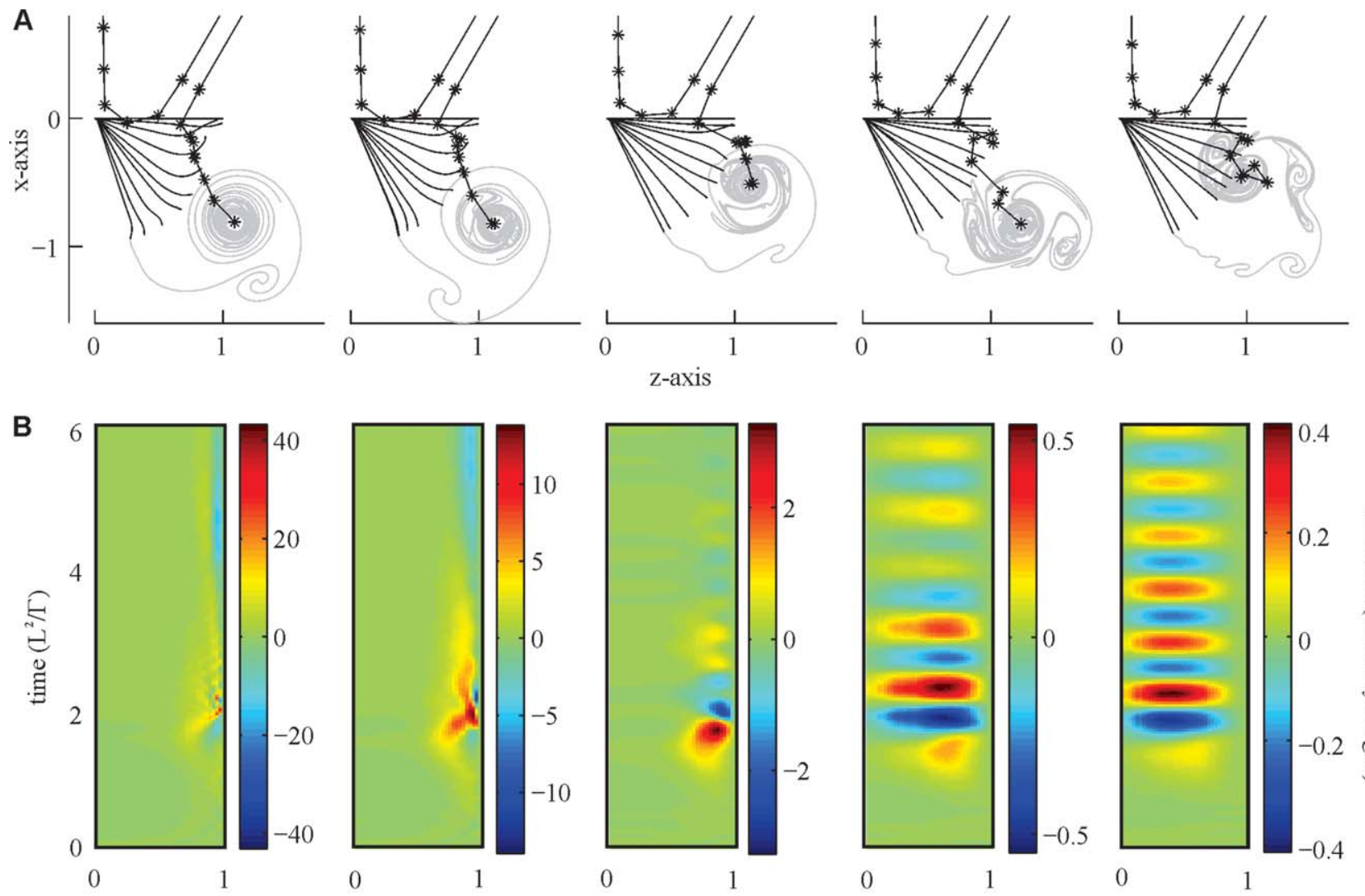

proportion of fin ray length

Fig. 8. Modeling of vortex perturbation impacting fin rays of different stiffness, ranging from very flexible (left) to very stiff (right). A: Snapshots of fin rays (solid lines) and vortex dipole pairs (asterisks) during impacts. The fin ray is held with a free hinge boundary condition at the origin in the $x-y$ plane, and the dipole pair is initially placed at the upper right of the frame, moving toward the fin ray. The asterisks corresponding to each member of the dipole pair are connected by solid lines, to show their trajectories in time as the pair dissociates. The flexural stiffness distributions are given by Eq. 2, with five different spatial decay constants, $\delta$ : (from left to right) $0.051,0.6,0.1,0.3,3$. These stiffness distributions are among those plotted in Figure 9A. B: Color maps of the curvatures of the fin rays over time, corresponding to the impacts in panel A. Each color map is below the corresponding impact snapshot. Units of time $\left(L^{2} / \Gamma\right)$ are equal to (fin ray length $)^{2} /$ (vortex dipole circulation) as described below Eq. 2 . The color bars indicate the curvature values in units of $1 /$ (fin ray length) and have different scales in each case.

the flexibility further caused a stronger attraction, with no apparent oscillation of curvature. Details of this attraction (and eventual collision), for a point vortex, are studied in Alben (2012).

Effect of segmentation. We also considered stiffness distributions which included the effect of segmentation, by adding a sinusoidal modulation to $B(s)$, as shown below Eq. 2. We simulated two values of $\delta, 0.1$ and 0.3 , and three segmentation wavenumbers $k(15,30$, and 60$)$. The $B(s)$ for $\delta=0.3$ and the three values of $k$ are shown by the upper three solid lines in Figure 10A; those for $\delta=0.1$ are the lower three solid lines. For $\delta=0.3$, snapshots of the segmented rays during impact are shown in Figure 10B; for $\delta=0.1$, the snapshots are shown in Figure 10C. In each case, the segmented fin rays moved very similarly to a smooth fin ray, plotted with dashed lines in panels $\mathrm{B}$ and $\mathrm{C}$. The $B(s)$ distributions for these smooth rays are shown by the dashed lines in Figure 10A. The smooth rays had a somewhat faster decay of $B(s)$ (smaller $\delta$ ) than their corresponding segmented rays, to compensate for the increased flexibility conferred by the sinusoidal modulation of the segmented rays. Figure 10D,E show the curvature maps corresponding to the segmented ray with the intermediate value of $k(30)$, and the corresponding smooth ray. For the segmented ray, the curvature is sharply peaked in bands which show the regions between the segments. For the unsegmented ray, the curvature is smooth, similar to the maps in Figure 8B. Taken together, Figure 10 shows that segmented and unsegmented rays can move very similarly during an impact. Therefore, the details of segmentation, such as how many segments and of which lengths occur on the fin ray, may be less important than the larger scale features of the flexibility distribution that segmentation confers. 
A

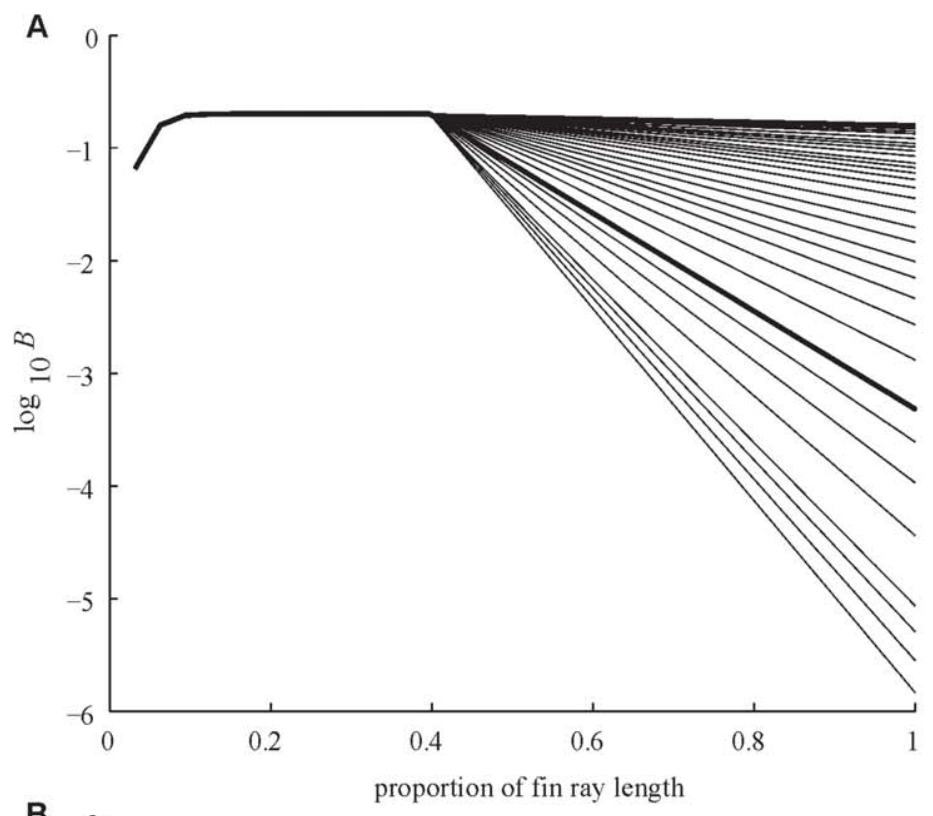

B

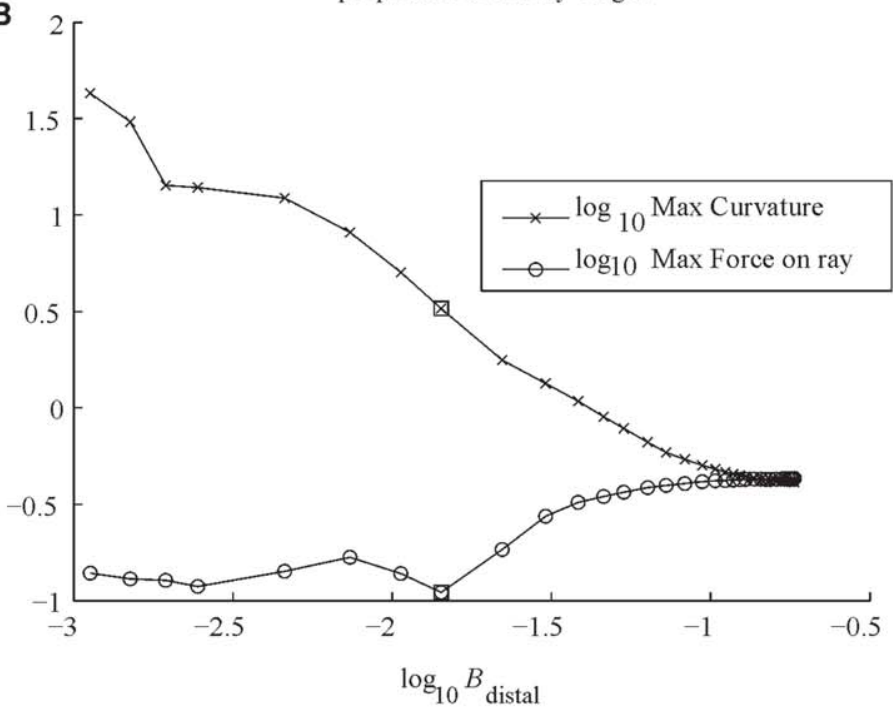

C

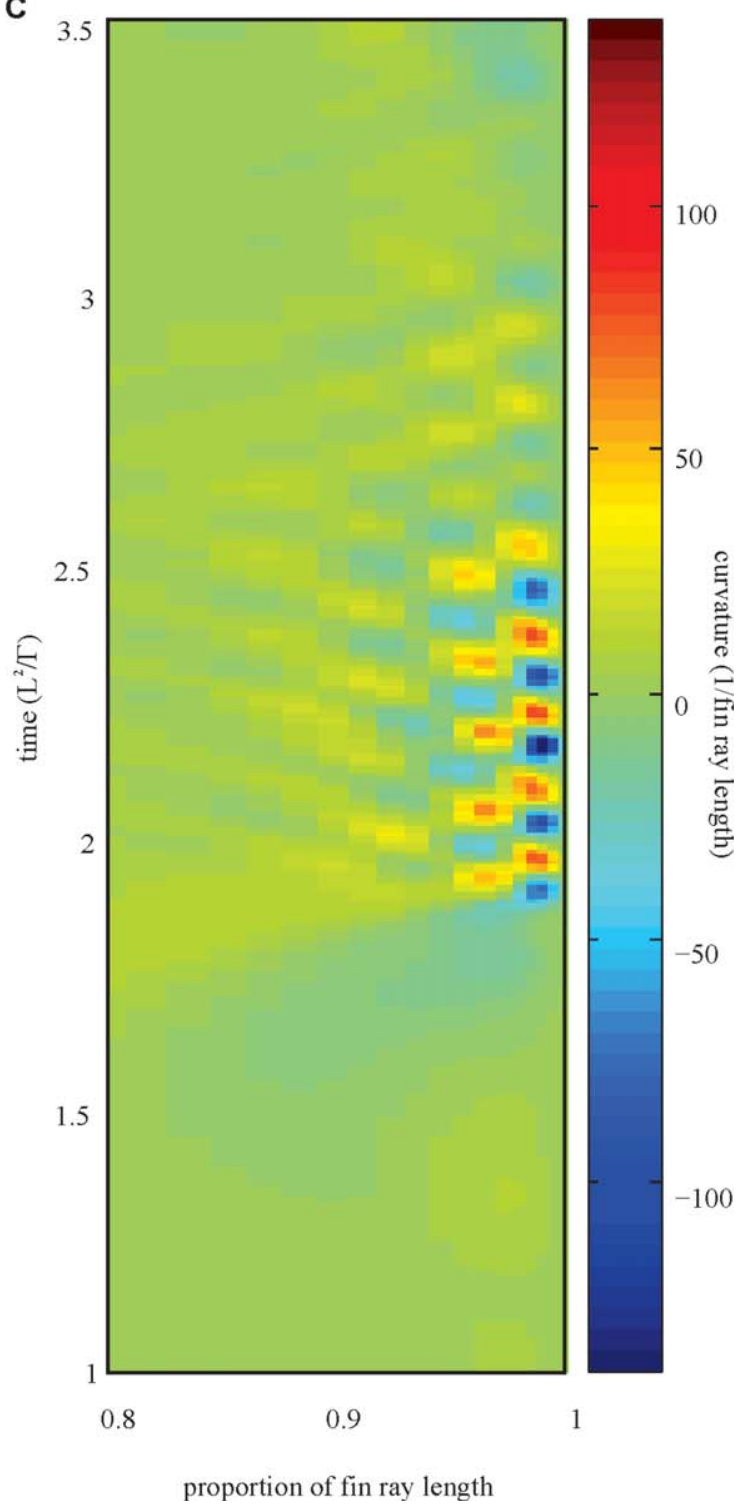

Fig. 9. A: Distributions of $B$ considered for unsegmented model. B: Maximum curvature and maximum total force magnitude during impact that the fluid exerts on rays of different stiffnesses. Values are plotted vs. the flexural stiffness of the ray at a location $2 / 3$ of the ray length from the base, and correspond to the stiffness distributions shown in A. The boxed values here correspond to the stiffness distribution with a thicker line in A, and correspond to $\delta=0.1$, close to that of biological specimens (Alben et al., 2007; Lauder et al., 2011). C: Curvature values near the tip (distal 20\%) for a very flexible ray ( $\delta=0.048$ in Eq. 2), illustrating localized rapid oscillation near impact. Units of time $\left(L^{2} / \Gamma\right)$ are equal to (fin ray length $)^{2} /($ vortex dipole circulation) as described below Eq. 2 . The color bar indicates the curvature values in units of $1 /$ (fin ray length).

\section{DISCUSSION}

The fin rays of ray-finned fishes are remarkable structures with a unique bilaminar design that allows active control of fin surface curvature and the ability to resist hydrodynamic loading on the fin. In this respect, the fins of actinopterygian fishes differ from insect wings and bird feathers and allow these fishes to potentially have fine control over the motion of their fins and interact in subtle ways with the surrounding fluid. Although recent years have seen an increase in the number of analyses of fin motion and in the mechanical properties of fins, and in vivo curvature values are now available for several taxa during swimming (Standen and Lauder, 2005; Taft et al., 2008), studies of fin ray mechanical properties have been limited to analyzing isolated rays removed from the fin (Alben et al., 2007; Lauder and Madden, 2007; Lauder et al., 2011; Taft and Taft, 2012). There remain a number of key aspects of fin functional design that need attention, including analysis of in vivo curvatures during different locomotor behaviors, and analysis 
A

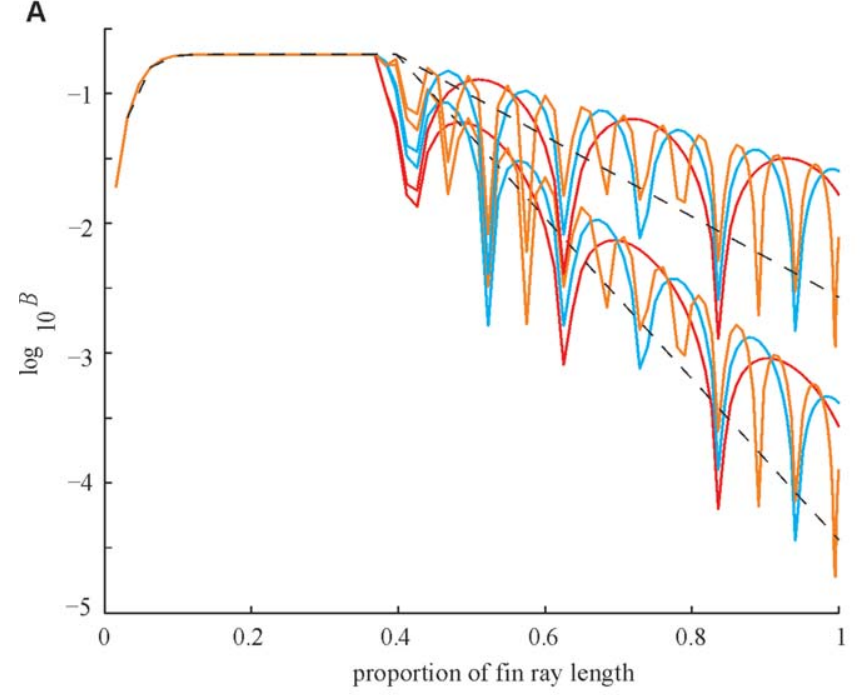

B
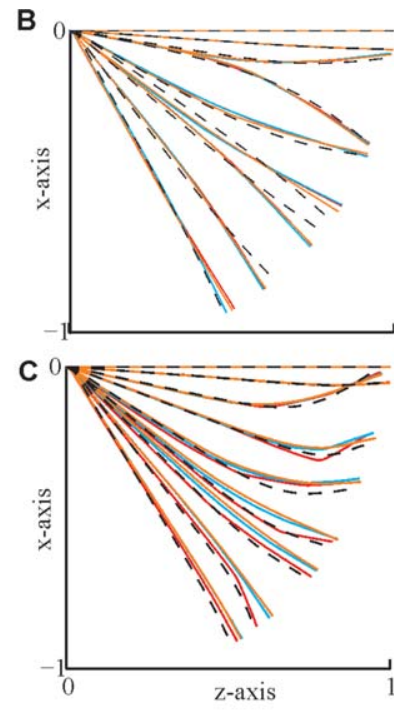

D
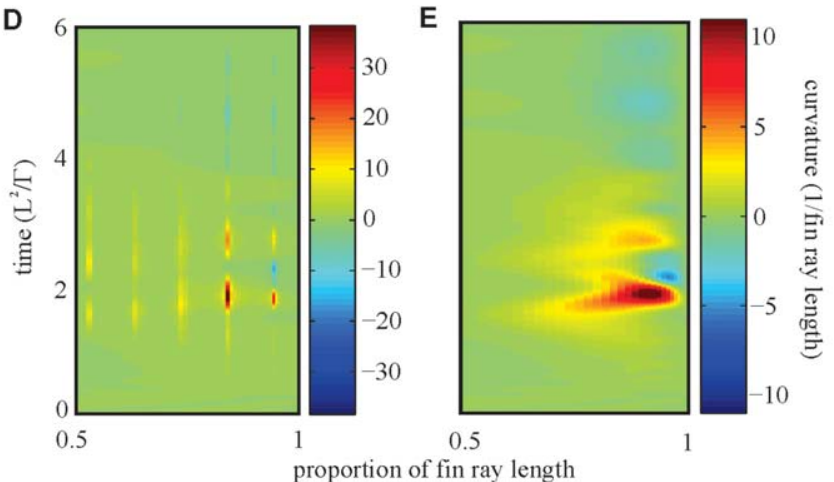

Fig. 10. Comparison of segmented and unsegmented fin ray models with flexural rigidity $(B)$ decay rates which give similar trajectories. A: For two $B$ decay rates ( $\delta=0.1$ and 0.3 in Eq. 2), $B$ distributions for fin rays with 3 (red), 6 (blue), and 12 (orange) segments are shown. B: Trajectory of stiffer segmented rays (3, 6, and 12 segments, solid lines) with decay rate $\delta=0.3$ (see below Eq. 2) during vortex dipole impact, together with an unsegmented ray (decay rate $\delta=0.14$, see below Eq. 2) giving a similar trajectory. C: Trajectory of flexible segmented rays (3, 6, and 12 segments, solid lines) with decay rate $\delta=0.1$ (see below Eq. 2) during vortex dipole impact, together with an unsegmented ray (decay rate $\delta=0.07$, see below Eq. 2) giving a similar trajectory. D: Values of curvature on the distal half of the segmented fin ray with $\delta=0.1$ and 6 segments in $\mathrm{C}$ over time. Units of time $\left(L^{2} / \Gamma\right)$ are equal to (fin ray length) ${ }^{2} /$ (vortex dipole circulation) as described below Eq. 2. E: Values of curvature on the distal half of the corresponding unsegmented fin ray in $\mathrm{C}$ (dashed line) over time. Units of time $\left(L^{2} / \Gamma\right)$ are equal to (fin ray length) ${ }^{2} /$ (vortex dipole circulation) as described below Eq. 2.

of how fin ray rays respond to environmental fluid perturbations.

In this article, we have studied the functional properties of the fin rays in ray-finned fishes by analyzing the curvature of the fins rays during a diversity of normal swimming behaviors including hovering and unsteady turns, and applied known perturbations to the fins in the form of a vortex ring aimed at the fin. This manner of perturbation allows us to apply a discrete, short-duration, wellcharacterized stimulus to the fin and observe the response (as opposed to directing a difficult-tocharacterize jet of fluid at the fin), and also to apply a computational model to the response of the fin rays. This model includes a fluid dynamic component allowing calculation of the movement of vortex rings toward the fin (another benefit of using a vortex ring stimulus), and also incorporates a mathematical model of the structural properties of the fin rays. The model thus allows us to estimate the in vivo stiffness of fin rays, and to compute the response that fin rays of different stiffnesses would have had to the same stimulus.

Perturbation of fin motion is an area of locomotor biomechanics in fishes that is still largely unexplored (although Webb (2004) has investigated the response of fish of different body shapes to the impact of fluid jets on the body), and yet this approach has the potential to clarify how fish fins function under conditions more similar to the natural environment than a laboratory flow tank.

\section{Curvature and Flexibility of Fish Pectoral Fin Rays}

Spanwise (along the length of the fin ray) curvature did not exceed $0.05 \mathrm{~mm}^{-1}$ during hovering or normal swimming and was at a maximum during 
turning $\left(0.065 \mathrm{~mm}^{-1}\right)$. The greatest pectoral fin curvatures observed were in the chordwise direction, reaching a maximum of $0.5 \mathrm{~mm}^{-1}$. These values fall within the range of all previously reported pectoral, dorsal, and anal fin curvature values (0.08-0.49 $\mathrm{mm}^{-1}$ ) for bluegill sunfish and sculpin (Standen and Lauder, 2005; Taft et al., 2008; Chadwell et al., 2012b; Taft and Taft, 2012). In the case of the sculpin, high curvatures in the ventral-most pectoral fin rays are an important functional specialization that permit station holding on a benthic substrate (Taft et al., 2008; Taft and Taft, 2012).

Maximum curvatures caused by vortex perturbation exceeded those seen under normal swimming conditions by several orders of magnitude; maximum curvature caused by perturbation was $9.38 \mathrm{~mm}^{-1}$. Therefore, fin rays are capable of much higher curvatures caused by buckling in response to the force of perturbation. This suggests that the curvatures seen during unrestrained swimming are minimized as a result of active stiffening to counteract hydrodynamic loading on the flexible fin rays (Lauder et al., 2011) and that studies of unperturbed swimming alone do not reveal the full extent of fin ray flexibility.

The flexural stiffness of bluegill sunfish pectoral fin rays is similar to that of highly flexible segmented crinoids $\left(392 \times 10^{-6} \mathrm{~N} \mathrm{~m}^{2}\right.$; Etnier, 2001), and falls within limits of other studies reporting fish flexural stiffness ranging from $0.6-1 \times 10^{-6} \mathrm{~N}$ $\mathrm{m}^{2}$ (McHenry et al., 1995; Long et al., 2002, 2006; Lauder et al., 2011). The Young's modulus of the unsegmented, proximal portion of the fin ray $(0.24-3.72 \mathrm{GPa})$ is between 2 and 6 times greater than that of the segmented, distal portion of the fin ray $(0.11-0.67 \mathrm{GPa})$ in bluegill sunfish (Lauder et al., 2011). Therefore, the incorporation of a segmented, distal portion of the fin ray offers a simple solution to producing a flexible structure from a rigid material, like bone.

Highly flexible fins allow fish to be more maneuverable by permitting active modulation of portions of their fins (Walker, 2004a,b; Lauder et al., 2006; Alben et al., 2007; Lauder and Madden, 2007). As a result, fish with flexible fins are able to redirect propulsive forces and effect low-radius turns about their center of mass (Drucker and Lauder, 1999, 2001a,b,2003; Lauder and Drucker, 2002, 2004).

\section{Functional Implications of Segmentation}

Examination of third pectoral fin rays from bluegill sunfish ranging in size from 6.1 to $16.9 \mathrm{~cm}$ TL showed that the proximal, unsegmented portion of the fin ray comprised about $40 \%$ of the total ray and the remainder of the fin ray (60\%) was segmented. This proportion was maintained through ontogeny despite the fact that fin ray growth is constant and occurs through the addition of additional segments to the distal end of the fin ray
(Johnson and Weston 1995). It may be important to maintain a rigid unsegmented portion of the fin ray more proximally in order to control adduction and abduction of the entire fin ray from the pectoral fin muscles at the fin ray base. A rigid proximal region of much less than $40 \%$ of the fin ray length may not exert enough control of the fin ray along its length to allow the distal region to accurately track motion of the base. In that case, the distal region of the fin ray could lag significantly behind the direction of fin motion during swimming.

Why should fish have flexible fin rays that require active stiffening and control, instead of fin rays that are entirely unsegmented and hence stiff along their entire length? There are at least three hypotheses that suggest why selective pressure may have favored flexible, segmented pectoral fin rays, and these three possibilities are not mutually exclusive: enhancing propulsion, bending on contact with the environment, and the ability to damp perturbations.

First, flexible fins have been shown to confer hydrodynamic advantage and, for certain stiffness values, to increase thrust forces in both robotic and computational models of fish fins (Lauder et al., 2006, 2011; Mittal et al., 2006; Alben et al., 2007; Lauder and Madden, 2007; Flammang, 2010; Tangorra et al., 2011). We have found that curvature values along individual fin rays during normal swimming, turning, and hovering behaviors are generally low, and suggest that there is an approximate balance between muscular forces acting at the fin base and hydrodynamic loading on the fin surface (Lauder and Madden, 2007). However, there are certainly changes in fin ray curvature that occur among behaviors. For example, we found that during steady swimming, fin ray 7 (in the center of the fin) yielded to the oncoming fluid against which it was moving, whereas during turning maneuvers, this ray was curved into oncoming flow. The fluid force producing bending during steady swimming could be due to a difference in fluid pressure on the upper and lower surface of the fin near ray 7 , resulting in the production of thrust during the abduction phase. Bluegill sunfish are capable of nearly continuous thrust production during pectoral fin swimming by cupping the pectoral fin during abduction (Lauder et al., 2006; Lauder and Madden, 2007). Turning maneuvers may be accomplished in part by redirecting these fluid dynamic forces through active control of the fin ray position.

Second, many ray-finned fishes use their pectoral fins to contact the environment around them. Bottom dwelling ray-finned fishes, like sculpins, use their flexible pectoral fins in order to hold onto the substrate and maintain position (Taft et al., 2008; Taft and Taft, 2012). It is also very likely that fishes are able to obtain sensory information by contacting their environment. Blind cavefish 
are known to follow walls by tapping with their pectoral fins, and it is assumed that this provides tactile information to the central nervous system (via sensory neurons within the fin) on wall location and perhaps distance to the wall (Voneida and Fish, 1984; Baker and Montgomery, 1999; Patton et al., 2010). Bending of fin rays as a result of contact may produce sensory information that would be useful to the fish for navigating complex environments (Hale and Williams, 2012; Williams et al., 2013). In order to grasp the substrate and activate mechanosensory inputs through bending stimuli, fin rays need to be flexible.

A third consideration is that the presence of segmentation in the distal portion of the fin allows for passive damping of fluid perturbations that might otherwise interfere with fin function or sensing during locomotion. Vortex perturbation of the pectoral fins of swimming fish, while causing major deformation to the fin ray at point of impact, had very little effect on more proximal portions of the fin ray driving the fin motion. Localized buckling between segments at the point of vortex impact was observed as small, focused epicenters of high curvature near the point of impact. Deforming perturbation forces absorbed by the fin ray were not transferred along segments to more basal fin ray regions. Using our model, we were able to demonstrate that more flexible fin rays are able to buckle at the point of impact allowing the vortex to roll off the fin with a minimal transfer of force, resulting in reduced perturbation to the fin ray along its length and at its base, potentially greatly reducing the effect of the perturbation on the frequency and amplitude of subsequent fin beats. Conversely, the stiffer fin rays experienced a "diving-board" type of oscillation from vortex impact and this resulted in disruptive deformation that spread along the length of the fin ray. Therefore, an advantage to having flexible fins may be to reduce the impact of hydrodynamic disturbances on the overall propulsive fluid mechanics of the fin, and such disturbances are common in natural settings.

\section{ACKNOWLEDGMENTS}

The authors would like to thank T. Gericke, D. Perl, and E. Youngerman for assistance in data collection and analysis, and to Lauder lab members who assisted with fish care responsibilities. Mark Ricco at Cornell University kindly performed the micro-CT scans of the fin rays.

\section{LITERATURE CITED}

Alben S. 2009. Simulating the dynamics of flexible bodies and vortex sheets. J Comput Phys 228:2587-2603.

Alben S. 2012. The attraction between a flexible filament and a point vortex. J Fluid Mech 697:481-503.
Alben S, Madden PGA, Lauder GV. 2007. The mechanics of active fin-shape control in ray-finned fishes. J Roy Soc Interface $4: 243-256$.

Alben S, Witt C, Baker TV, Anderson E, Lauder GV. 2012. Dynamics of freely swimming flexible foils. Phys Fluids A 24: 051901.

Baker CF, Montgomery JC. 1999. The sensory basis of rheotaxis in the blind Mexican cavefish, Astyanax fasciatus. J Comp Physiol A 184:519-527.

Chadwell BA, Standen EM, Lauder GV, Ashley-Ross MA. 2012a. Median fin function during the escape response of bluegill sunfish (Lepomis macrochirus). I: Fin-ray orientation and movement. J Exp Biol 215:2869-2880.

Chadwell BA, Standen EM, Lauder GV, Ashley-Ross MA. 2012b. Median fin function during the escape response of bluegill sunfish (Lepomis macrochirus). II: Fin-ray curvature. J Exp Biol 215:2881-2890.

Cohen L, Dean M, Shipov A, Atkins A, Monsonego-Ornan E, Shahar R. 2012. Comparison of structural, architectural, and mechanical aspects of cellular andacellular bone in two teleost fish. J Exp Biol 215:1983-1993.

Drucker EG, Lauder GV. 1999. Locomotor forces on a swimming fish: Three-dimensional vortex wake dynamics quantified using digital particle image velocimetry. J Exp Biol 202: 2393-2412.

Drucker EG, Lauder GV. 2001a. Locomotor function of the dorsal fin in teleost fishes: Experimental analysis of wake forces in sunfish. J Exp Biol 204:2943-2958.

Drucker EG, Lauder GV. 2001b. Wake dynamics and fluid forces of turning maneuvers in sunfish. J Exp Biol 204:431-442.

Drucker EG, Lauder GV. 2003. Function of pectoral fins in rainbow trout: Behavioral repertoire and hydrodynamic forces. J Exp Biol 206:813-826.

Etnier SA. 2001. Flexural and torsional stiffness in multijointed biological beams. Biol Bull 200:1-8.

Flammang BE. 2010. Functional morphology of the radialis muscle in shark tails. J Morphol 271:340-352.

Geerlink PJ, Videler JJ. 1987. The relation between structure and bending properties of teleost fin rays. Neth J Zool 37: 59-80.

Hale ME, Williams R. 2012. Pectoral fins as sensors: Spatial distribution of sensory input to the pectoral fins of bluegill sunfish. Integr Comp Biol 52:e71.

Hedrick TL. 2008. Software techniques for two- and threedimensional kinematic measurements of biological and biomimetic systems. Bioinsp Biomim 3:034001-034006.

Johanson Z, Burrow C, Warren A, Garvey J. 2005. Homology of fin lepidotrichia in osteichthyan fishes. Lethaia 38:27-36.

Krasny R. 1986. Desingularization of periodic vortex sheet rollup. J Comput Physics 65:292-313.

Lauder GV, Drucker EG. 2002. Forces, fishes, and fluids: Hydrodynamic mechanisms of aquatic locomotion. News Physiol Sci 17:235-240.

Lauder GV, Drucker EG. 2004. Morphology and experimental hydrodynamics of fish fin control surfaces. IEEE J Ocean Engin 29:556-571.

Lauder GV, Madden PGA. 2007. Fish locomotion: Kinematics and hydrodynamics of flexible foil-like fins. Exper Fluids 43: 641-653.

Lauder GV, Madden PGA, Mittal R, Dong H, Bozkurttas M. 2006. Locomotion with flexible propulsors I: Experimental analysis of pectoral fin swimming in sunfish. Bioinsp Biomim 1:S25-S34.

Lauder G, Madden PGA, Tangorra J, Anderson E, Baker TV. 2011. Bioinspiration from fish for smart material design and function. Smart Mat Struct 20:094014 (094013p).

Long JH, Jr., Koob TJ, Irving K, Combie K, Engel V, Livingston N, Lammert A, Schumacher J. 2006. Biomimetic evolutionary analysis: Testing the adaptive value of vertebrate tail stiffness in autonomous swimming robots. J Exp Biol 209: 4732-4746.

Long JH, Koob-Emunds M, Sinwell B, Koob TJ. 2002. The notochord of hagfish Myxine glutinosa: Visco-elastic properties 
and mechanical functions during steady swimming. J Exp Biol 205:3819-3831.

McHenry MJ, Pell CA, Long JA. 1995. Mechanical control of swimming speed: Stiffness and axial wave form in undulating fish models. J Exp Biol 198:2293-2305.

Mittal R, Dong H, Bozkurttas M, Lauder GV, Madden PGA 2006. Locomotion with flexible propulsors: II. Computational modeling of pectoral fin swimming in sunfish. Bioinspir Bio$\operatorname{mim}$ 2006:S35-S41.

Moss ML. 1963. The biology of acellular teleost bone. Ann New York Acad Sci 109:337-350.

Patton P, Windsor S, Coombs S. 2010. Active wall following by Mexican blind cavefish (Astyanax mexicanus). J Comp Physiol A 196:853-867.

Rasband WS. 1997-2012. ImageJ, U.S. National Institutes of Health, Bethesda, Maryland, USA, Available at: http://imagej. nih.gov/ij/.

Standen EM, Lauder GV. 2005. Dorsal and anal fin function in bluegill sunfish Lepomis macrochirus: Three-dimensional kinematics during propulsion and maneuvering. J Exp Biol 208 $2753-2763$

Taft NK, Lauder GV, Madden PGA. 2008. Functional regionalization of the pectoral fin of the benthic longhorn sculpin during station holding and swimming. J Zool 276:159-167.
Taft NK, Taft BN. 2012. Functional implications of morphological specializations among the pectoral fin rays of benthic longhorn sculpins. J Exp Biol 215:2703-2710.

Tangorra J, Gericke T, Lauder GV. 2011. Learning from the fins of ray-finned fish for the propulsors of unmanned undersea vehicles. Mar Tech Soc J 45:1-18.

Videler JJ. 1993. Fish Swimming. London: Chapman and Hall. $260 \mathrm{p}$.

Voneida TJ, Fish SE. 1984. Central nervous system changes related to the reduction of visual input in a naturally blind fish (Astyanax hubbsi). Am Zool 24:775-782.

Walker JA. 2004a. Dynamics of pectoral fin rowing in a fish with an extreme rowing stroke: The threespine stickleback (Gasterosteus aculeatus). J Exp Biol 207:1925-1939.

Walker JA. 2004b. Kinematics and performance of maneuvering control surfaces in teleost fishes. IEEE J Ocean Eng 29: 572-584.

Webb PW. 2004. Response latencies to postural disturbances in three species of teleostean fishes. J Exp Biol 207: 955-961.

Williams R, Neubarth NL, Hale ME. 2013. The function of fin rays as proprioceptive sensors in fish. Nature Comm 4:1729. doi: $10.1038 /$ ncomms2751. 